\title{
UNDERSTANDING INDONESIA'S MACROECONOMIC DATA: WHAT DO WE KNOW AND WHAT ARE THE IMPLICATIONS?
}

\author{
Susan Sunila Sharma ${ }^{1}$, Lutzardo Tobing ${ }^{2}$, Prayudhi Azwar ${ }^{3}$ \\ ${ }^{1}$ Department of Finance \& Centre for Financial Econometrics, Deakin Business School, Australia. \\ Email: s.sharma@deakin.edu.au \\ ${ }^{2}$ Bank Indonesia Institute, Bank Indonesia, Jakarta, Indonesia. Email: lutzardo@bi.go.id \\ ${ }^{3}$ Bank Indonesia Institute, Bank Indonesia, Jakarta, Indonesia. Email: yudhi@bi.go.id
}

\begin{abstract}
Unit root properties of macroeconomic data are important for both econometric modeling and policymaking. The form of variables (whether they are a unit root process) helps determine the correct econometric model. Equally, the form of variables helps explain how they react to shocks (both internal and external). Macroeconomic time-series data are often at the forefront of shock analysis and econometric modeling. There is a growing research emphasis on Indonesia using time-series data; yet, there is limited understanding of the data characteristics and shock response of these data. Using an extensive dataset comprising 33 macroeconomic time-series variables, we provide an informative empirical analysis of unit root properties of this data. We find that, regardless of data frequency, empirical evidence of unit roots is mixed. Some data series respond quickly to shocks while others take more time. Almost all macroeconomic data suffer from structural breaks. We draw implications from these findings.
\end{abstract}

Keywords: Unit root; Macroeconomic data; Structural breaks; Shocks; Econometric modeling. JEL Classification: C5; E1.

Article history:

Received : July 1, 2018

Revised : October 20, 2018

Accepted : October 20, 2018

Available online : October 31, 2018

https://doi.org/10.21098/bemp.v21i2.967 


\section{INTRODUCTION}

Unit Root Properties (URP) have implications for how applied researchers and policymakers interpret and use data. URP assists in understanding the form of data. There are two forms data can take, either stationary or non-stationary. In simple terms, stationary time-series data have mean, variance, and co-variance that do not change over time. By comparison, a non-stationary series is best characterized as one whose mean, variance, and co-variance change over time. Precise knowledge of the form of the time-series data is important, because when its form is stationary, this implies that shocks will have short-term (or temporary) effects. On the other hand, a non-stationary series implies that shocks have long-term or permanent effects on the variable. This knowledge has policy implications because policymakers need to understand the form of variables to deduce how they will react to policy changes and/or shocks.

The second advantage from understanding the form of variables has roots in econometric modeling. Applied researchers are constrained by theory in modeling data. Theory also tends to dictate the form in which variables need to be modeled. There are many examples of this. Two are offered here for demonstration. First, consider the Purchasing Power Parity (PPP) hypothesis, which holds that prices equalize across countries, meaning that any price differences on a good/service in any two like countries should be stationary for PPP to hold; see Narayan (2006a). Second, the popular efficient market hypothesis argues that asset prices (such as stock prices) should be stationary (see Narayan and Smyth, 2007).

So great has been the influence of unit roots pioneered by Nelson and Plosser (1982) - considering the need to understand the shock reaction of variables and the form in which they enter econometric modeling, as discussed above-that there is a separate literature on new tests for unit roots; see also Perron (1989), which marks the starting point for research based on structural break(s). In other words, researchers have focused attention on developing more robust unit root tests that can offer greater precision when testing for the precise form of the data. Two avenues for improvement noted recently are important to highlight. Endogenous structural break treatment has a notable history in unit root testing. However, while the tests became available following Lee and Strazicich (2003), subsequent work (see, for instance, Narayan and Popp, 2010) took issue with the precision in estimating the break dates themselves, because accurate identification of breaks has implications for precise understanding of the form of the data (Narayan and Popp, 2010). More recent work (Narayan and Liu, 2015; Narayan, Liu and Westerlund, 2016) takes issue with the fact that when modeling for unit roots, it is not only structural breaks that are important, but also the role of a time trend and data heteroskedasticity can be equally important in delivering an unbiased understanding of the data.

Macroeconomic data are also important for Indonesia. Several studies analyze Indonesian macroeconomic data via testing different relations. For instance, Amir, Asafu-Adjaye, and Ducpham (2013) examine the impact of Indonesia's income tax reforms on various macroeconomic variables, namely real Gross Domestic Product (GDP), real private consumption, real investment, real government consumption, real exports, real imports, consumer price index, 
GDP price index, and average real wage. Dutu (2016) examines economic growth slowdowns in Indonesia. Hsing (2012) examines the impact of macroeconomic forces and external shocks on Indonesia's real output. Chowdhury, Uddin, and Anderson (2018) examine the influence of monetary and fiscal policy variables on the market and firm-level liquidity of eight emerging stock markets in Asia. Tanuwidjaja and Choy (2006) examine the role of Indonesian central bank credibility in achieving an inflation target. Hadiwibowo and Komatsu (2011) examine the macroeconomic trilemma and international capital flows under several financial structures in Indonesia. Djuranovik (2014) develops a model of the term structure of interest rates in Indonesia to create a link between the yield curve and macroeconomic fundamentals, namely real activity, inflation, and interest rate. Sowmya and Prasanna (2018) examine interaction between the yield curve and macroeconomic factors of Asian economies. Such studies and future research would benefit from greater understanding of the importance of unit root tests.

Returning to the idea of understanding the form of the variable, what started off as instrumental knowledge in using macroeconomic data spread quickly to other fields of research where shocks were relevant in understanding how variables respond to them. The unit root idea, for instance, was popularized in Narayan and Smyth (2007) in a time-series setting and extended to a panel data setting in Narayan, Narayan, and Smyth (2008). In tourism economics, the idea was introduced by Narayan (2005a,b) and in health economics by Narayan (2006b). The main message of these studies is that unit root evidence is important to understanding the nature and impact of shocks not only with macroeconomic data (see Section II), but also with other time-series data where shocks are relevant, such as in energy, tourism, and health.

This paper proceeds as follows. Section II reviews the literature on the presence of unit root in macroeconomic data. Section III discusses our data and results. Finally, Section IV sets forth concluding remarks.

\section{THE LITERATURE}

This section provides a feel for the importance of understanding the unit root behavior of macroeconomic data. We choose selected studies from this literature that we believe best offers a snapshot of the work done on unit roots devoted to macroeconomic data.

Table 1 summarizes selected literature on unit roots. We believe that these studies provide a reasonable representation of the literature and the features that characterize this literature. Let us identify these features more precisely. First, note from Column 2 that unit root tests of macroeconomic data are conducted at different data frequencies (annually, weekly, quarterly, and monthly), although most work seems to use annual data followed by monthly data. The dominance of annual data is expected given that, for most countries, macroeconomic data (over time) is available only annually. One issue arising from this concerns robustness. The question arises of whether the evidence on unit root data is frequency-dependent. We address this by undertaking a unit root test on both annual and monthly data. A caveat here is that one ends up with different start dates when using 
higher frequency data. The implication is that a strict comparison of the unit root hypothesis across data frequencies is impossible. However, the advantage is that we have some results that we can consider, depending on policy objectives.

The second feature of the literature, which can be read from Column 3, is that a wide range of macroeconomic data are utilized in unit root tests. The most popular data series seem to be GDP, inflation, and exchange rate; the highest number of variables used is around 14. Our study presents an extensive unit root analysis focusing on Indonesia-our sample includes 33 annual time-series data and 31 monthly time-series data. This represents a first comprehensive analysis of unit root testing of macroeconomic data.

The third feature concerns the econometric approach taken to test the unit root hypothesis. There are several points to note here. First, early studies seem to use tests without structural breaks. These studies are complemented by papers that address the unit root issue with structural breaks. Second, recent studies employ panel data models. Thus, the literature has progressed from time-seriesbased methods to panel data-based methods for testing the unit root hypothesis. We position our study within the popular structural break unit root testing methodology.

The final feature concerns the evidence on unit root. At best, the evidence appears mixed. Two trends are notable, however. First, panel data models offer greater evidence of stationarity. One reason for this is the gain in power to reject the unit root null that results from an increase in sample size when data is pooled across cross-sections and over time. Second, time-series models that accommodate structural break(s) offer greater evidence of stationarity (evidence against the unit root null hypothesis). These factors have implications for how one should approach unit root testing in macroeconomic data. We employ structural break unit roots tests within a time-series setting.

\section{DATA AND RESULTS}

Time-series data are used for unit root testing. A total of 31 monthly and 33 annual time-series macroeconomic variables for Indonesia are employed in this study. A complete list of variables is provided in Tables 2 (monthly series) and 3 (annual series). In summary, our dataset has three bond yield variables (separated by maturity), four interbank interest rate variables (separated by maturity), nine financial variables (business confidence index, capital value added, cash return index, dividend yield, Dow Jones stock index, market capitalization to GDP, Jakarta stock exchange Islamic index, price-to-earnings ratio, stock return index), and 17 monetary/trade-related variables (CPI, deposit rate, industrial production, composite index, exchange rate, export goods, export index, import goods, import index, industrial production, lending rate, M1, M2, producer price index, foreign exchange reserves, unemployment, and wholesale price index). All data are obtained from the Global Financial Database. 


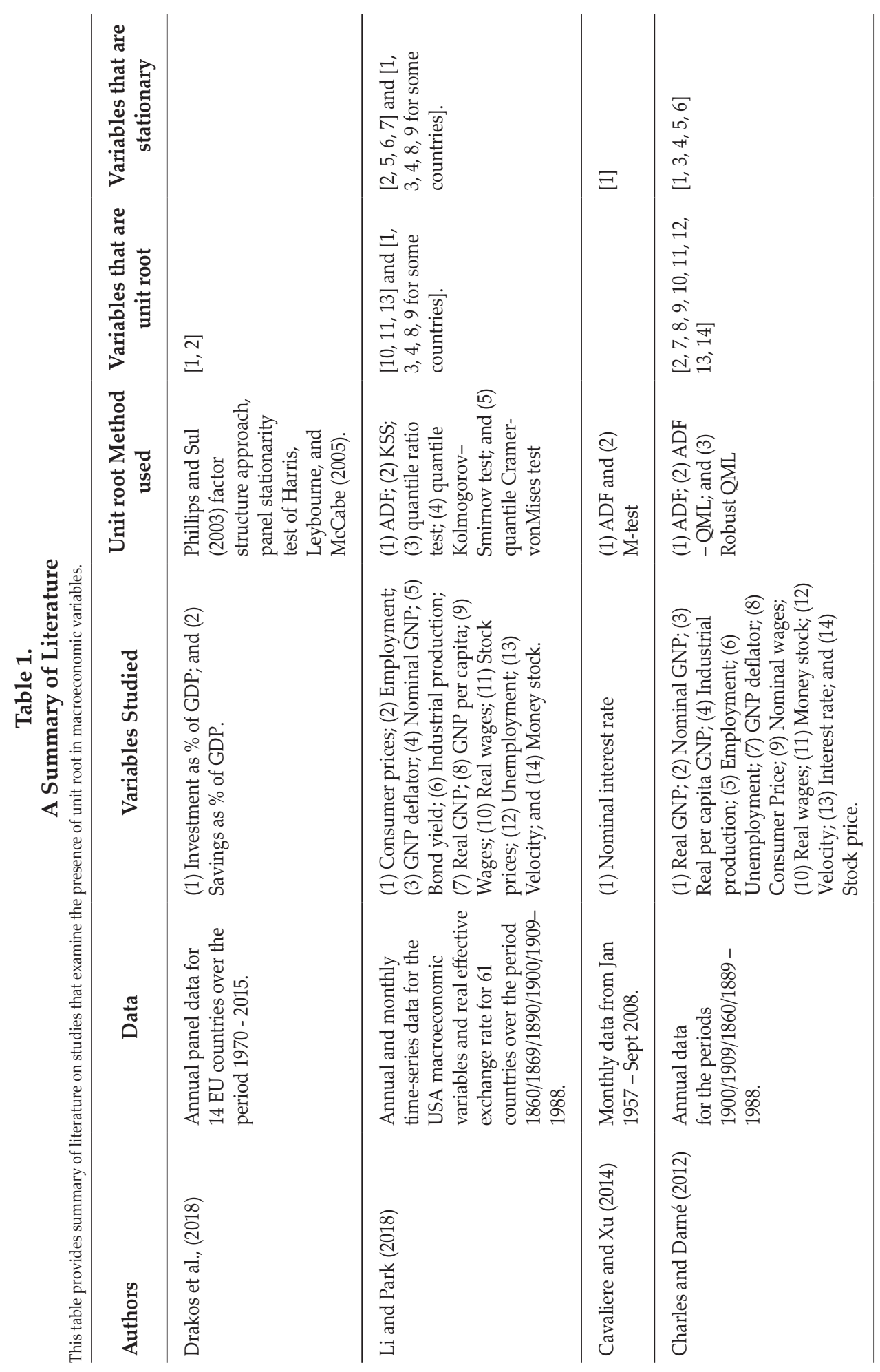




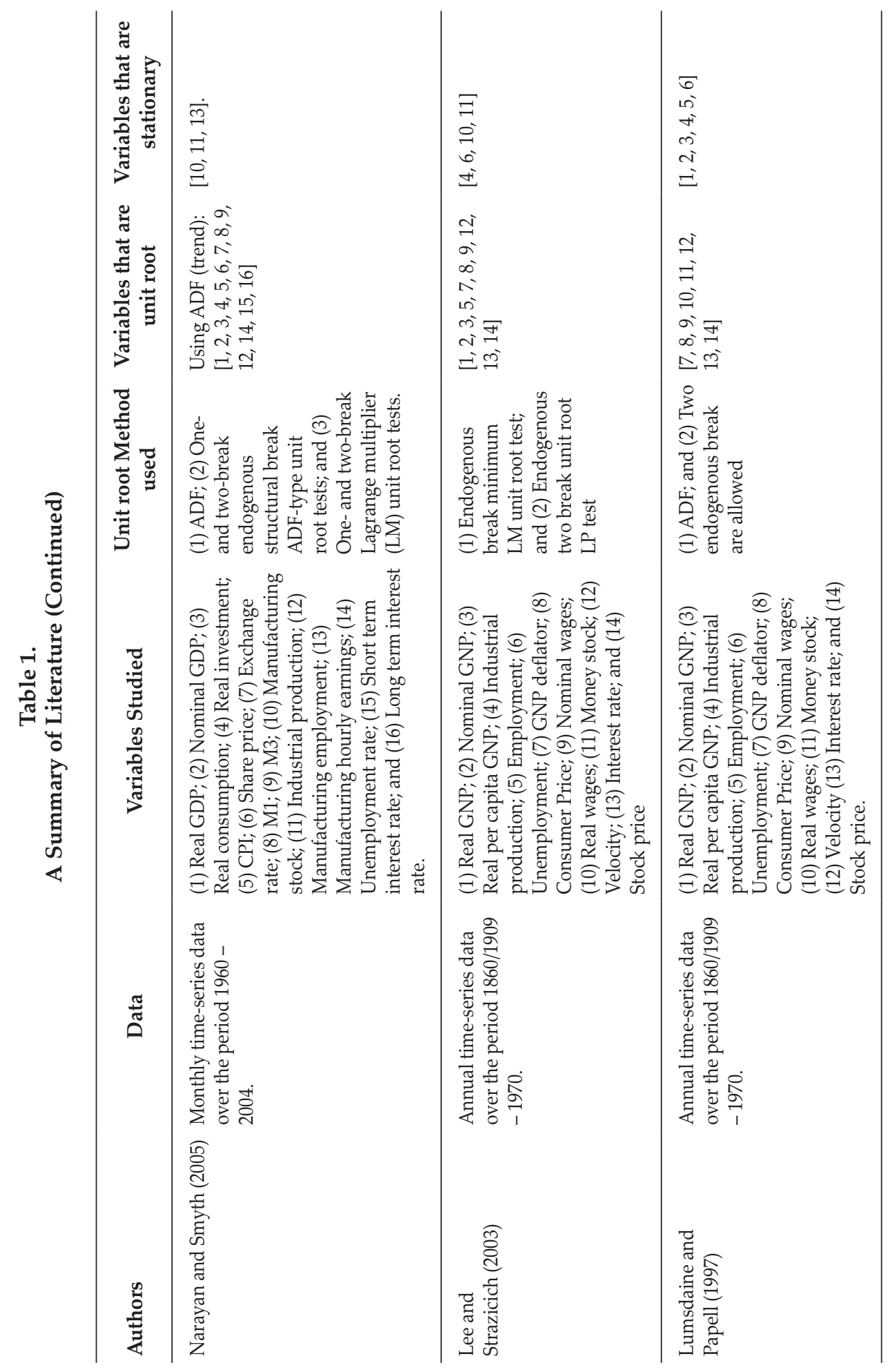




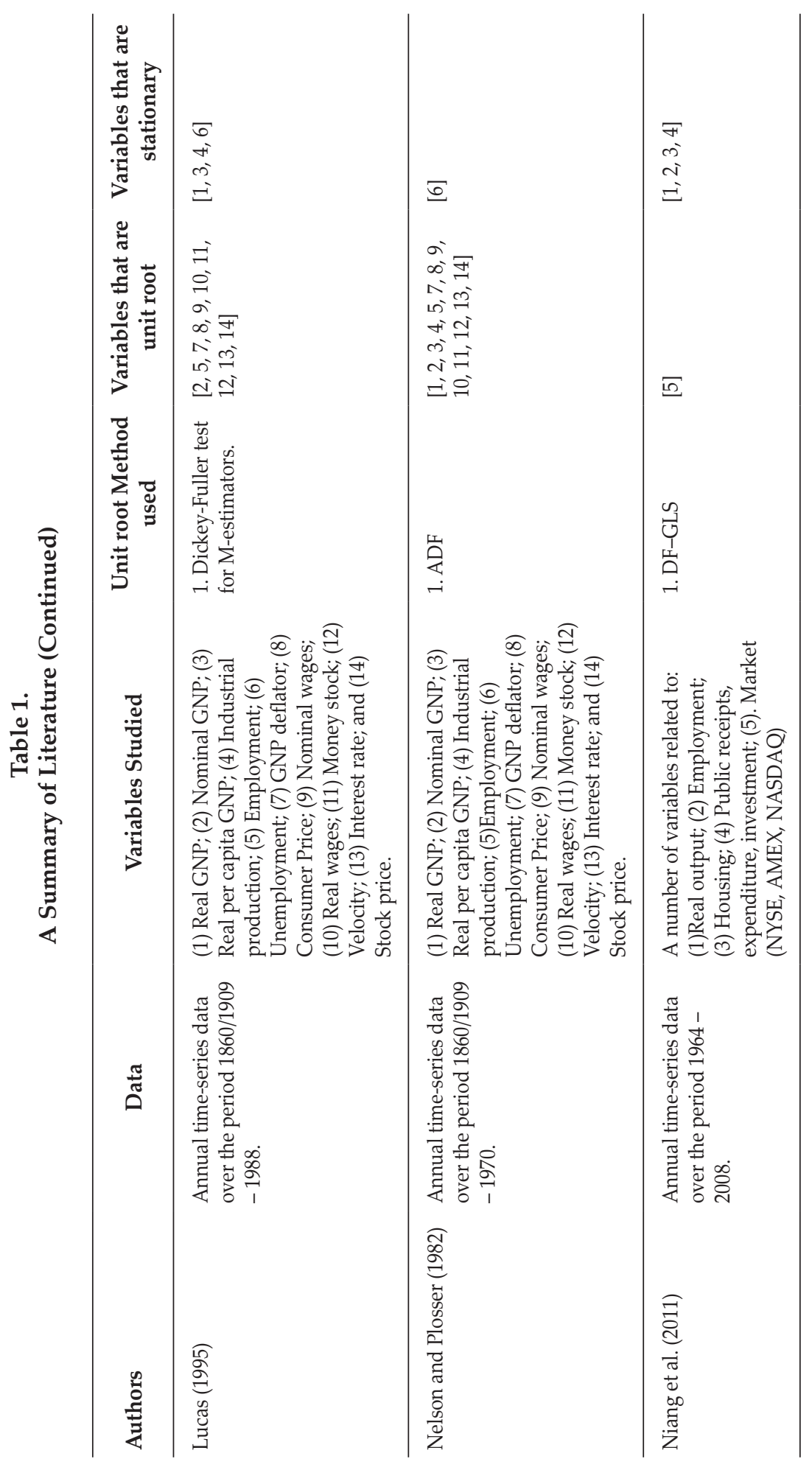




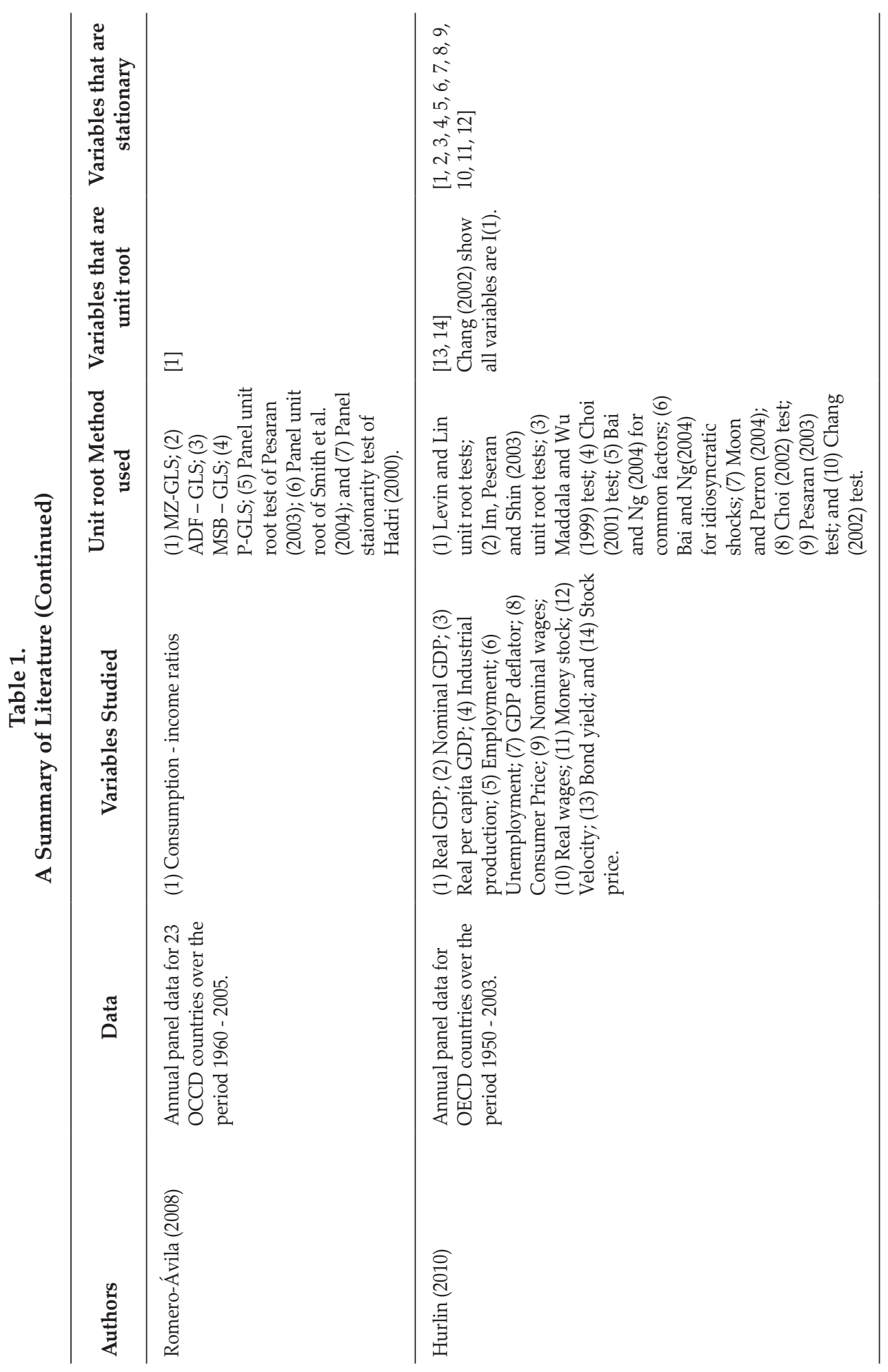




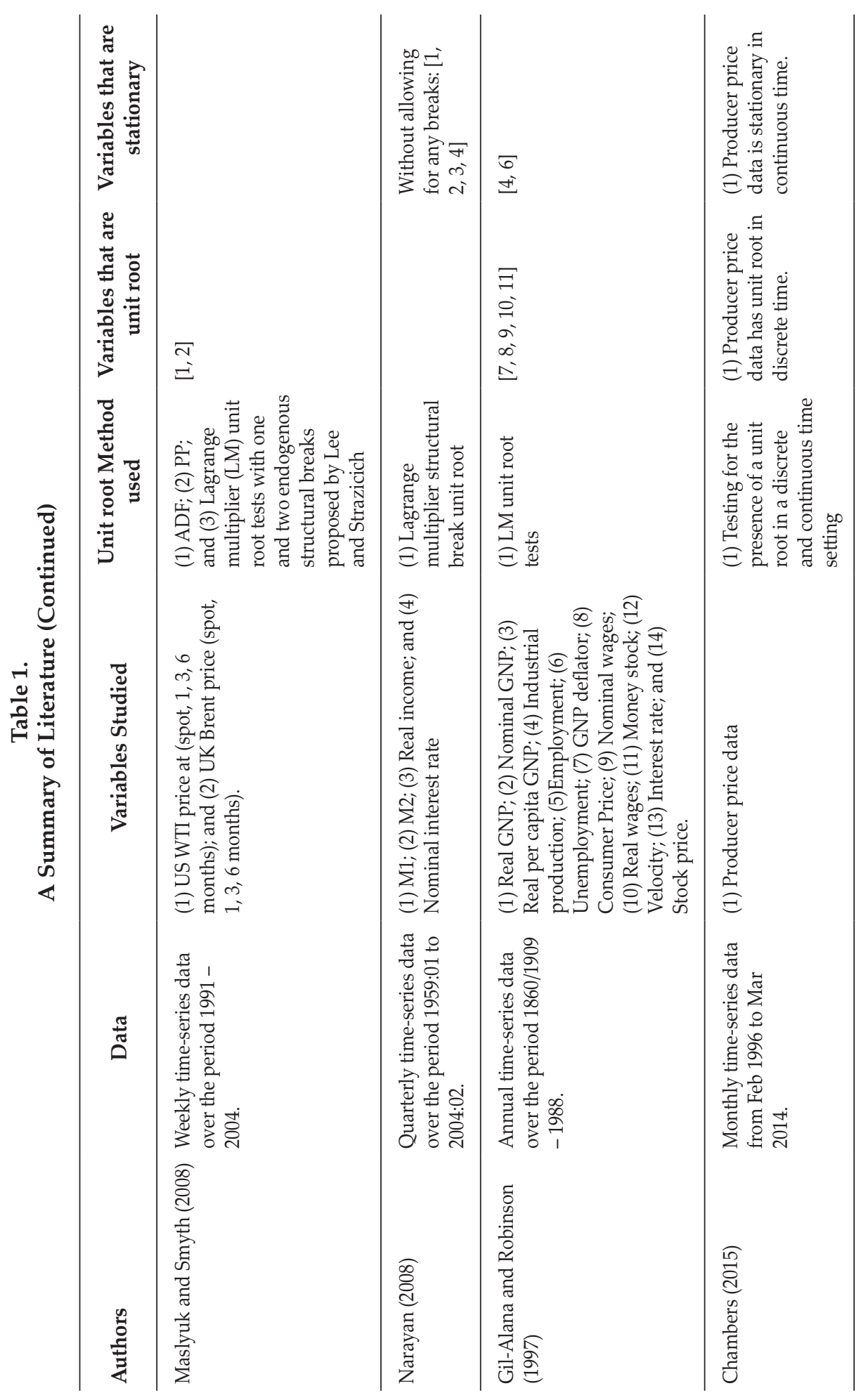




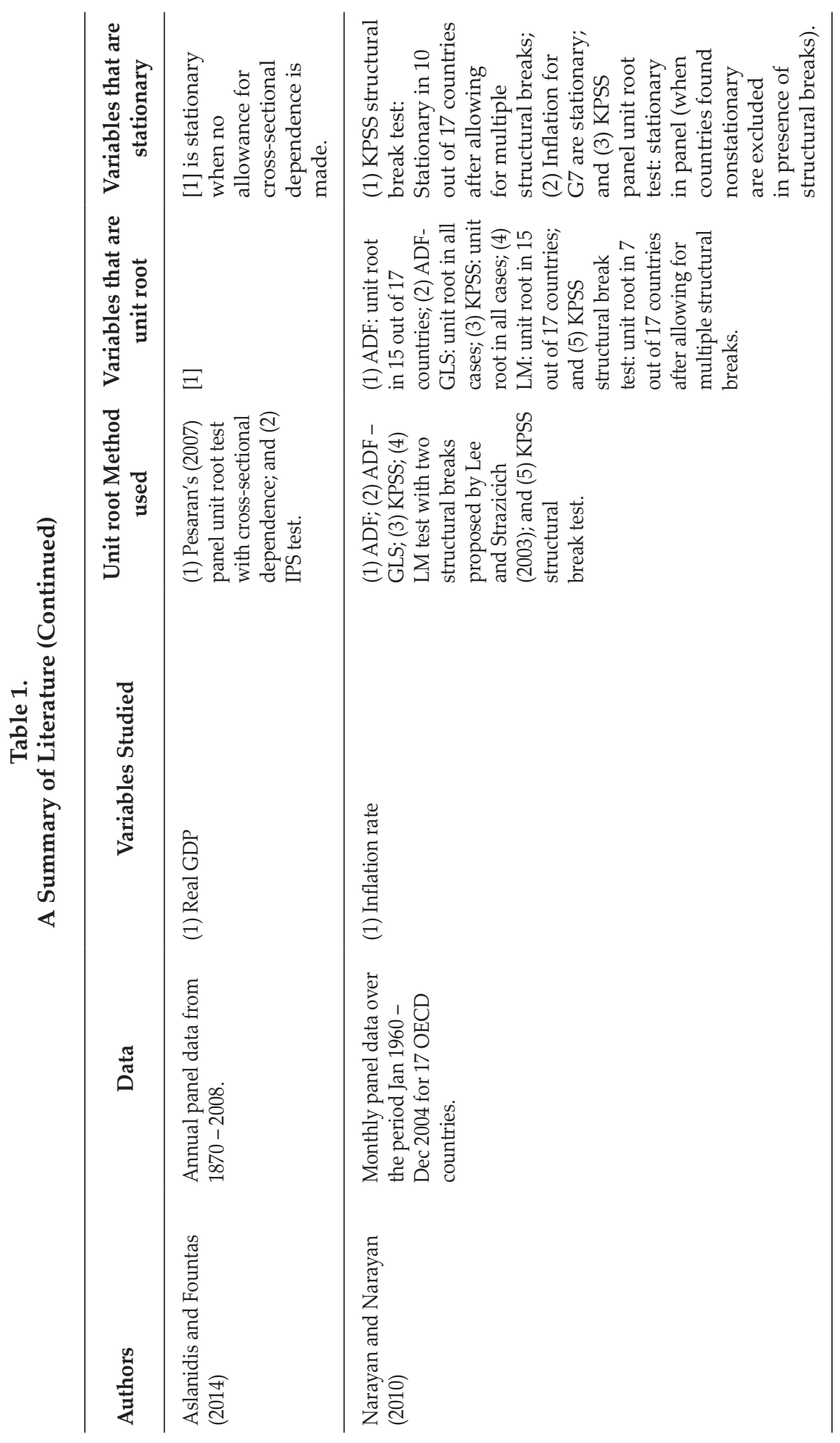




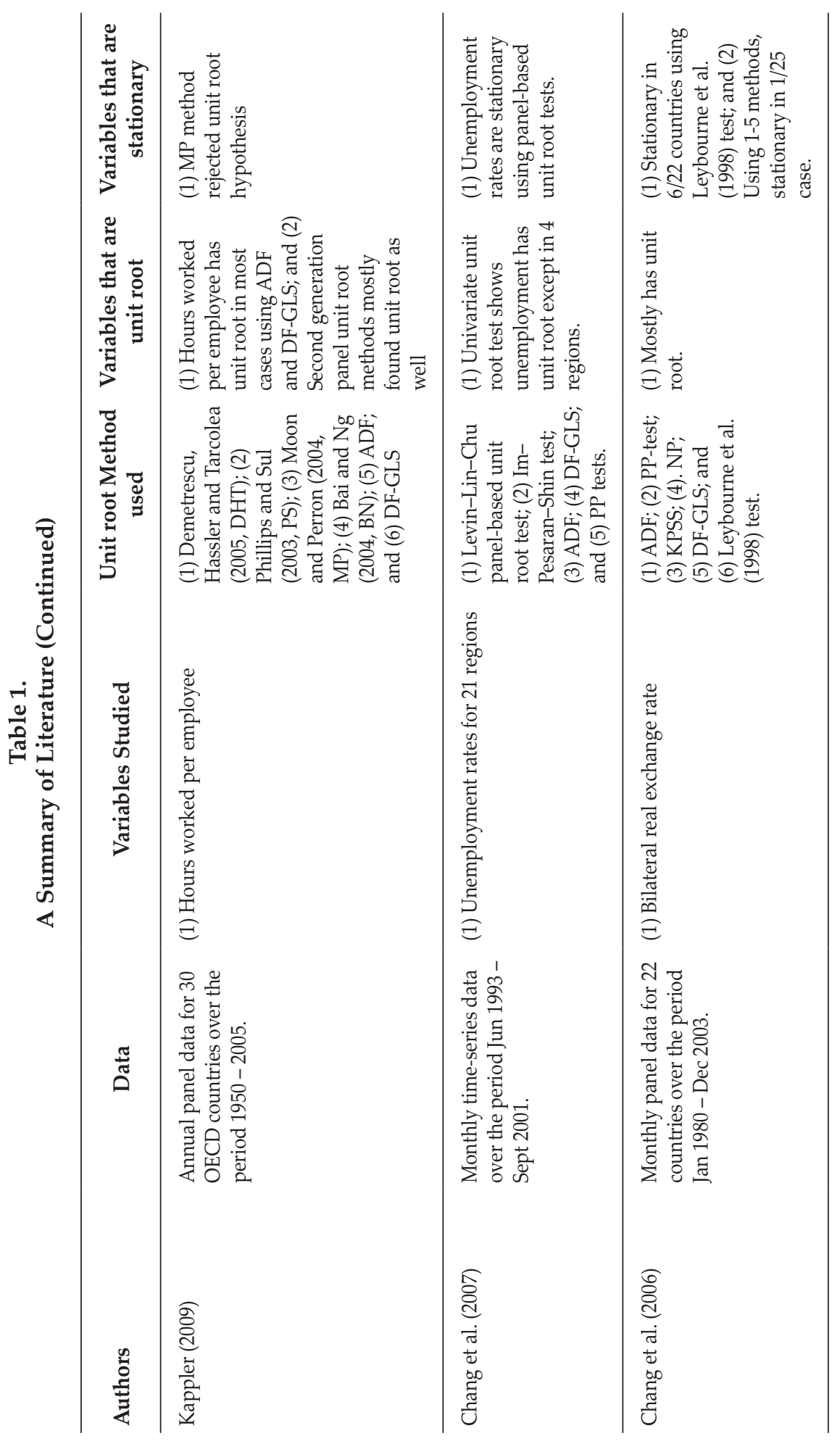




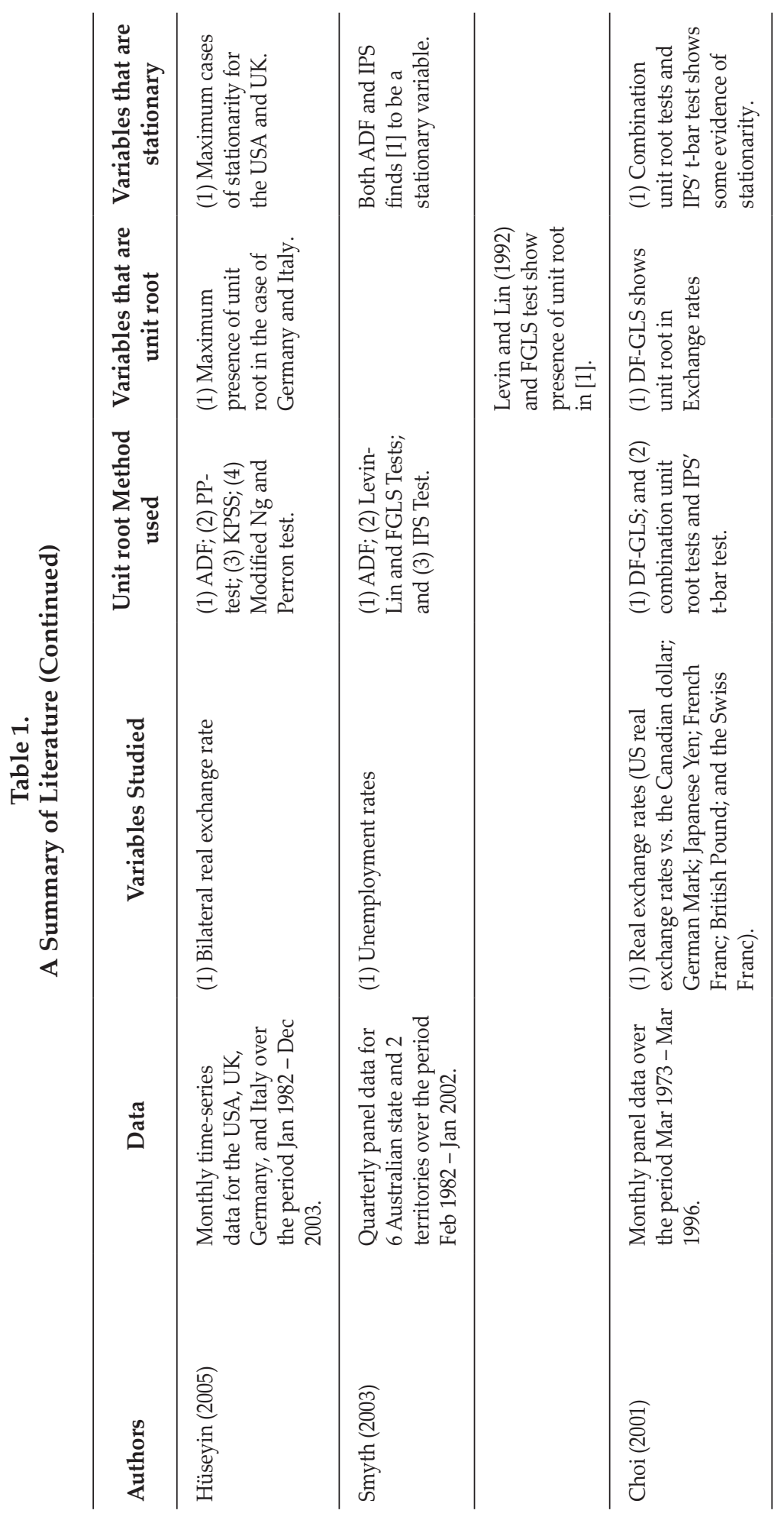


Table 2.

\section{Descriptive Statistics of Monthly Data}

This table presents descriptive statistics for monthly data. Thirty-one data series are considered, and Column 3 contains the sample period for each series followed by the number of observations (Obs.) in the sample. The mean, Standard Deviation (SD), skewness, Jarque-Bera (JB) test coefficient and its respective p-values are presented in Columns 5 to 9 , respectively. The JB test examines the null hypothesis of a normal distribution.

\begin{tabular}{|c|c|c|c|c|c|c|c|c|}
\hline No. & Series & Sample Period & Obs. & Mean & Std. Dev. & Skewness & Jarque-Bera & $p$-value \\
\hline 1 & Bond Yield, 3 Year & 2009:05-2018:06 & 110 & 1.814 & 0.178 & -0.672 & 9.194 & 0.010 \\
\hline 2 & Bond Yield, 5 Year & 2009:05-2018:06 & 110 & 1.952 & 0.182 & -0.453 & 5.342 & 0.069 \\
\hline 3 & Bond Yield, 10 Year & 2009:05-2018:06 & 110 & 2.019 & 0.170 & -0.125 & 0.794 & 0.672 \\
\hline 4 & $\begin{array}{l}\text { Business Confidence } \\
\text { Index }\end{array}$ & 2002:01-2017:12 & 190 & 4.602 & 0.010 & -1.526 & 97.560 & 0.000 \\
\hline 5 & Capital Value Traded & 1990:01-2018:05 & 341 & 11.288 & 1.334 & -0.235 & 16.770 & 0.000 \\
\hline 6 & Cash Return Index & 1989:12-2018:06 & 343 & 4.480 & 1.122 & -0.513 & 34.768 & 0.000 \\
\hline 7 & Composite Index & 1983:03-2018:06 & 424 & 6.582 & 1.365 & -0.073 & 16.025 & 0.000 \\
\hline 8 & $\begin{array}{l}\text { Consumer Confidence } \\
\text { Index }\end{array}$ & 2001:04-2017:12 & 201 & 4.601 & 0.013 & -1.062 & 56.344 & 0.000 \\
\hline 9 & CPI Inflation & 1967:01-2018:06 & 618 & 2.630 & 1.615 & -0.333 & 32.348 & 0.000 \\
\hline 10 & Deposit Rate & 1974:04-2016:07. & 508 & 2.421 & 0.495 & 0.364 & 16.186 & 0.000 \\
\hline 11 & Dividend Yield & 1990:11-2018:06 & 332 & 0.598 & 0.651 & -2.934 & 1850.536 & 0.000 \\
\hline 12 & Exchange Rate & 1876:01-2018:06 & 1710 & -0.629 & 6.495 & 0.519 & 259.035 & 0.000 \\
\hline 13 & Dow Jones Stock Index & 1992:01-2018:06 & 318 & 5.982 & 0.836 & 0.152 & 33.685 & 0.000 \\
\hline 14 & Export Goods & 1961:01-2018:05 & 689 & 9.772 & 1.846 & -0.617 & 65.459 & 0.000 \\
\hline 15 & Export Index & 1991:01-2018:05 & 329 & -0.304 & 0.289 & 0.040 & 20.510 & 0.000 \\
\hline 16 & $\begin{array}{l}\text { GFD Market } \\
\text { Capitalisation of GDP }\end{array}$ & 1995:01-2018:05 & 281 & -7.049 & 1.534 & 0.672 & 58.232 & 0.000 \\
\hline 17 & Import Goods & 1960:01-2018:06 & 701 & 9.426 & 1.856 & -0.370 & 46.696 & 0.000 \\
\hline 18 & Import Index & 1991:01-2018:05 & 329 & -0.295 & 0.324 & -0.641 & 25.998 & 0.000 \\
\hline 19 & $\begin{array}{l}\text { Indonesia } 1 \text { Month } \\
\text { Interbank Interest Rate } \\
\text { (JIBOR) }\end{array}$ & 1990:01-2018:06 & 342 & 2.357 & 0.546 & 0.914 & 73.150 & 0.000 \\
\hline 20 & $\begin{array}{l}\text { Indonesia } 3 \text { Month } \\
\text { Interbank Interest Rate } \\
\text { (JIBOR) }\end{array}$ & 1993:12-2018:06 & 295 & 2.340 & 0.526 & 0.996 & 64.297 & 0.000 \\
\hline 21 & $\begin{array}{l}\text { Indonesia } 6 \text { Month } \\
\text { Intebank Interest Rate } \\
\text { (JIBOR) }\end{array}$ & 1991:01-2018:06 & 330 & 2.382 & 0.478 & 0.779 & 39.274 & 0.000 \\
\hline 22 & $\begin{array}{l}\text { Indonesia } 12 \text { Month } \\
\text { Intebank Interest Rate } \\
\text { (JIBOR) }\end{array}$ & 1997:03-2018:06 & 256 & 2.334 & 0.484 & 1.127 & 66.305 & 0.000 \\
\hline 23 & $\begin{array}{l}\text { Industrial Production } \\
\text { Volume }\end{array}$ & 1991:12-2018:04 & 317 & 12.579 & 0.224 & 0.208 & 8.340 & 0.015 \\
\hline 24 & $\begin{array}{l}\text { Jakarta Stock Exchange } \\
\text { Islamic Index }\end{array}$ & 2000:07-2018:06 & 216 & 5.700 & 0.861 & -0.715 & 26.511 & 0.000 \\
\hline 25 & $\begin{array}{l}\text { Lending Rate for } \\
\text { Working Capital }\end{array}$ & 1986:03-2016:08 & 366 & 2.860 & 0.275 & 0.316 & 10.954 & 0.004 \\
\hline 26 & M1-Money Supply & 2008:01-2018:04 & 124 & 13.550 & 0.366 & -0.150 & 8.081 & 0.018 \\
\hline 27 & M2-Money supply & 200:801-2018:04 & 124 & 14.965 & 0.374 & -0.234 & 9.233 & 0.010 \\
\hline
\end{tabular}


Table 2.

Descriptive Statistics of Monthly Data (Continued)

\begin{tabular}{|c|c|c|c|c|c|c|c|c|}
\hline No. & Series & Sample Period & Obs. & Mean & Std. Dev. & Skewness & Jarque-Bera & $p$-value \\
\hline 28 & Price to Earnings Ratio & 1990:01-2018:06 & 342 & 2.813 & 0.342 & 0.049 & 32.162 & 0.000 \\
\hline 29 & $\begin{array}{l}\text { Producer Price Index } \\
\text { Excluding Oil }\end{array}$ & 1971:01-2016:04 & 544 & 2.604 & 1.575 & -0.200 & 26.700 & 0.000 \\
\hline 30 & Stock Return Index & 1988:01-2018:06 & 366 & 7.637 & 1.286 & 0.153 & 22.583 & 0.000 \\
\hline 31 & $\begin{array}{l}\text { Total Foreign } \\
\text { Exchange Reserves } \\
\text { (exclude Gold) }\end{array}$ & 1971:01-2018:06 & 570 & 9.383 & 1.659 & -0.478 & 24.609 & 0.000 \\
\hline
\end{tabular}

Table 3.

Descriptive Statistics of Yearly Data

This table presents descriptive statistics for yearly data. Thirty-three data series are considered, and Column 3 contains the sample period for each series followed by the number of observations (Obs.) in the sample. The mean, Standard Deviation (SD), skewness, Jarque-Bera (JB) test coefficient and its respective p-values are presented in Columns 5 to 9, respectively. The JB test examines the null hypothesis of a normal distribution.

\begin{tabular}{|c|c|c|c|c|c|c|c|c|}
\hline No & Series & Sample Period & Obs. & Mean & Std. Dev. & Skewness & Jarque-Bera & $p$-value \\
\hline 1 & Capital Value Traded & 1977-2017 & 41 & 9.119 & 3.556 & -0.665 & 4.758 & 0.093 \\
\hline 2 & Cash Return Index & $1989-2017$ & 29 & 4.443 & 1.164 & -0.494 & 2.929 & 0.231 \\
\hline 3 & Composite Index & 1977-2017 & 41 & 6.305 & 1.448 & 0.174 & 2.463 & 0.292 \\
\hline 4 & CPI & $1960-2016$ & 57 & 1.626 & 3.295 & -1.647 & 36.827 & 0.000 \\
\hline 5 & CPI Inflation & $1948-2017$ & 70 & -0.351 & 5.297 & -0.955 & 12.002 & 0.002 \\
\hline 6 & Deposit Rate & 1974-2017 & 44 & 2.406 & 0.502 & 0.514 & 1.974 & 0.373 \\
\hline 7 & Dividend Yield & $1990-2017$ & 28 & 0.585 & 0.696 & -2.858 & 132.257 & 0.000 \\
\hline 8 & Dow Jones Stock Index & 1992-2017 & 26 & 5.991 & 0.849 & 0.113 & 2.676 & 0.262 \\
\hline 9 & Exchange Rate & $1818-2017$ & 200 & -2.170 & 6.002 & 1.058 & 41.992 & 0.000 \\
\hline 10 & Export Goods & $1946-2017$ & 72 & 9.102 & 2.191 & -0.251 & 5.507 & 0.064 \\
\hline 11 & Export Goods and Services & $1990-2017$ & 28 & 13.221 & 1.256 & -0.393 & 2.339 & 0.311 \\
\hline 12 & Export Index & 1991-2017 & 27 & -0.299 & 0.284 & 0.071 & 1.951 & 0.377 \\
\hline 13 & GDP-Deflator Inflation & $1961-2015$ & 55 & 2.758 & 1.100 & 0.970 & 8.970 & 0.011 \\
\hline 14 & GDP-Deflator & $1960-2015$ & 56 & 1.671 & 2.166 & -0.278 & 2.359 & 0.307 \\
\hline 15 & $\begin{array}{l}\text { GFD Market Capitalisation } \\
\text { of GDP }\end{array}$ & 1993-2017 & 25 & -6.875 & 1.591 & 0.612 & 4.731 & 0.094 \\
\hline 16 & Nominal GDP & 1951-2017 & 67 & 9.383 & 6.128 & -0.850 & 9.208 & 0.010 \\
\hline 17 & Real GDP & $1870-2017$ & 148 & 13.421 & 1.263 & 0.634 & 14.674 & 0.001 \\
\hline 18 & Import Goods & $1946-2017$ & 72 & 8.847 & 2.111 & -0.048 & 5.574 & 0.062 \\
\hline 19 & Import Goods and Services & $1990-2017$ & 28 & 13.221 & 1.256 & -0.393 & 2.339 & 0.311 \\
\hline 20 & Import Index & 1991-2017 & 27 & -0.280 & 0.307 & -0.458 & 2.140 & 0.343 \\
\hline 21 & $\begin{array}{l}\text { Indonesia } 1 \text { Month } \\
\text { Interbank Interest Rate } \\
\text { (JIBOR) }\end{array}$ & $1990-2017$ & 28 & 2.366 & 0.503 & 0.523 & 1.281 & 0.527 \\
\hline 22 & $\begin{array}{l}\text { Indonesia } 3 \text { Month } \\
\text { Interbank Interest Rate } \\
\text { (JIBOR) }\end{array}$ & 1993-2017 & 25 & 2.361 & 0.506 & 0.708 & 2.202 & 0.332 \\
\hline 23 & $\begin{array}{l}\text { Indonesia } 6 \text { Month } \\
\text { Interbank Interest Rate } \\
\text { (JIBOR) }\end{array}$ & 1991-2017 & 27 & 2.383 & 0.471 & 0.728 & 2.732 & 0.255 \\
\hline
\end{tabular}


Table 3.

Descriptive Statistics of Yearly Data (Continued)

\begin{tabular}{|c|c|c|c|c|c|c|c|c|}
\hline No & Series & Sample Period & Obs. & Mean & Std. Dev. & Skewness & Jarque-Bera & $p$-value \\
\hline 24 & $\begin{array}{l}\text { Indonesia } 12 \text { Month } \\
\text { Interbank Interest Rate } \\
\text { (JIBOR) }\end{array}$ & 1997-2017 & 21 & 2.341 & 0.474 & 0.889 & 2.814 & 0.245 \\
\hline 25 & $\begin{array}{l}\text { Industrial Production } \\
\text { Volume }\end{array}$ & 1991-2017 & 27 & 12.575 & 0.232 & 0.203 & 0.753 & 0.686 \\
\hline 26 & $\begin{array}{l}\text { Lending Rate for Working } \\
\text { Capital }\end{array}$ & $1986-2017$ & 32 & 2.832 & 0.286 & 0.296 & 0.881 & 0.644 \\
\hline 27 & Price to Earnings Ratio & $1990-2017$ & 28 & 2.805 & 0.307 & -0.429 & 1.332 & 0.514 \\
\hline 28 & $\begin{array}{l}\text { Producer Price Index } \\
\text { Excluding Oil }\end{array}$ & 1971-2017 & 47 & 2.740 & 1.607 & -0.209 & 2.468 & 0.291 \\
\hline 29 & Stock Return Index & $1987-2017$ & 31 & 7.573 & 1.356 & 0.072 & 1.378 & 0.502 \\
\hline 30 & $\begin{array}{l}\text { Total Foreign Exchange } \\
\text { Reserves (exclude Gold) }\end{array}$ & 1971-2017 & 47 & 9.410 & 1.651 & -0.473 & 1.947 & 0.378 \\
\hline 31 & Total Reserve & $1960-2015$ & 56 & 8.334 & 2.585 & -0.768 & 5.835 & 0.054 \\
\hline 32 & Unemployment & $1973-2017$ & 35 & 1.711 & 0.858 & 2.621 & 201.509 & 0.000 \\
\hline 33 & Wholesale Price Index & $1971-2016$ & 46 & 2.662 & 1.604 & -0.207 & 2.265 & 0.322 \\
\hline
\end{tabular}

A plot of the annual time-series data is available in Figure 1. Tables 2 and 3 show descriptive statistics based on monthly and annual time-series data, respectively. Given the time-series nature of the data, we note from both these tables the start data. Not all series have lengthy data. For example, some series, like exchange rate, have data going as far back as 1876. Inflation and deposit rate data are available from the 1960s and 1970s, respectively, while for other series much smaller data samples are available. Details are found in Columns 2 and 3 of these tables. Thus, data series have different start dates. This is dictated entirely by data availability.

\section{Figure 1. A Plot of Annual Time-Series Data}

This figure plots annual time-series data for 33 variables. Full variable description is given in Appendix Table A1. The time-span of each variable is dependent on data availability and is explicitly noted in Tables 2-3.

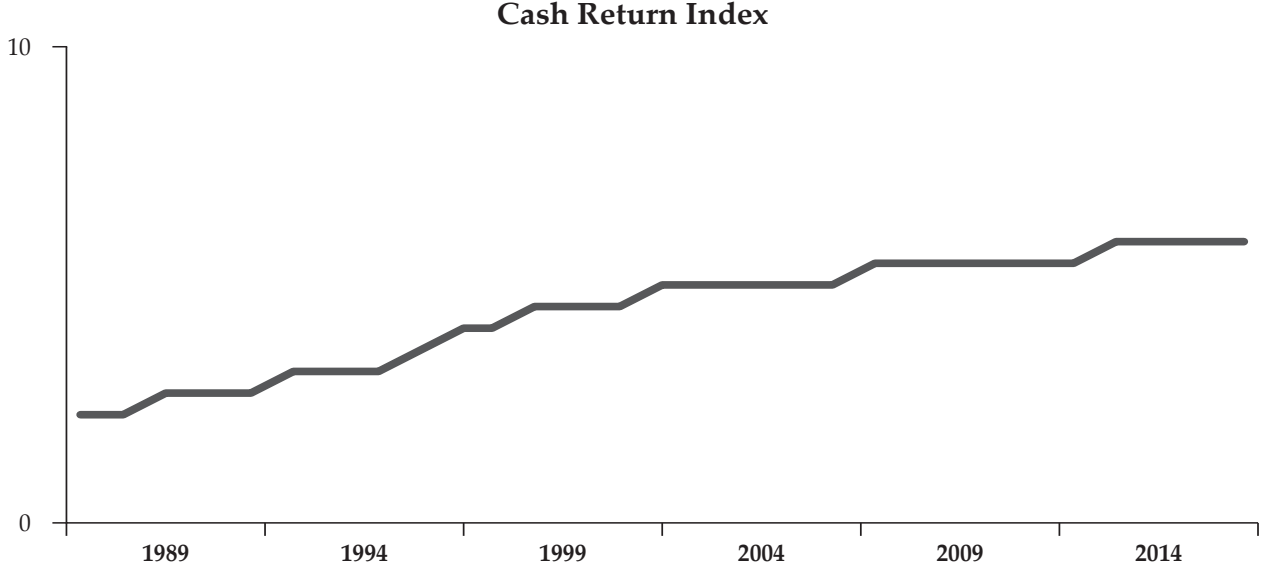


Figure 1. A Plot of Annual Time-Series Data (Continued)
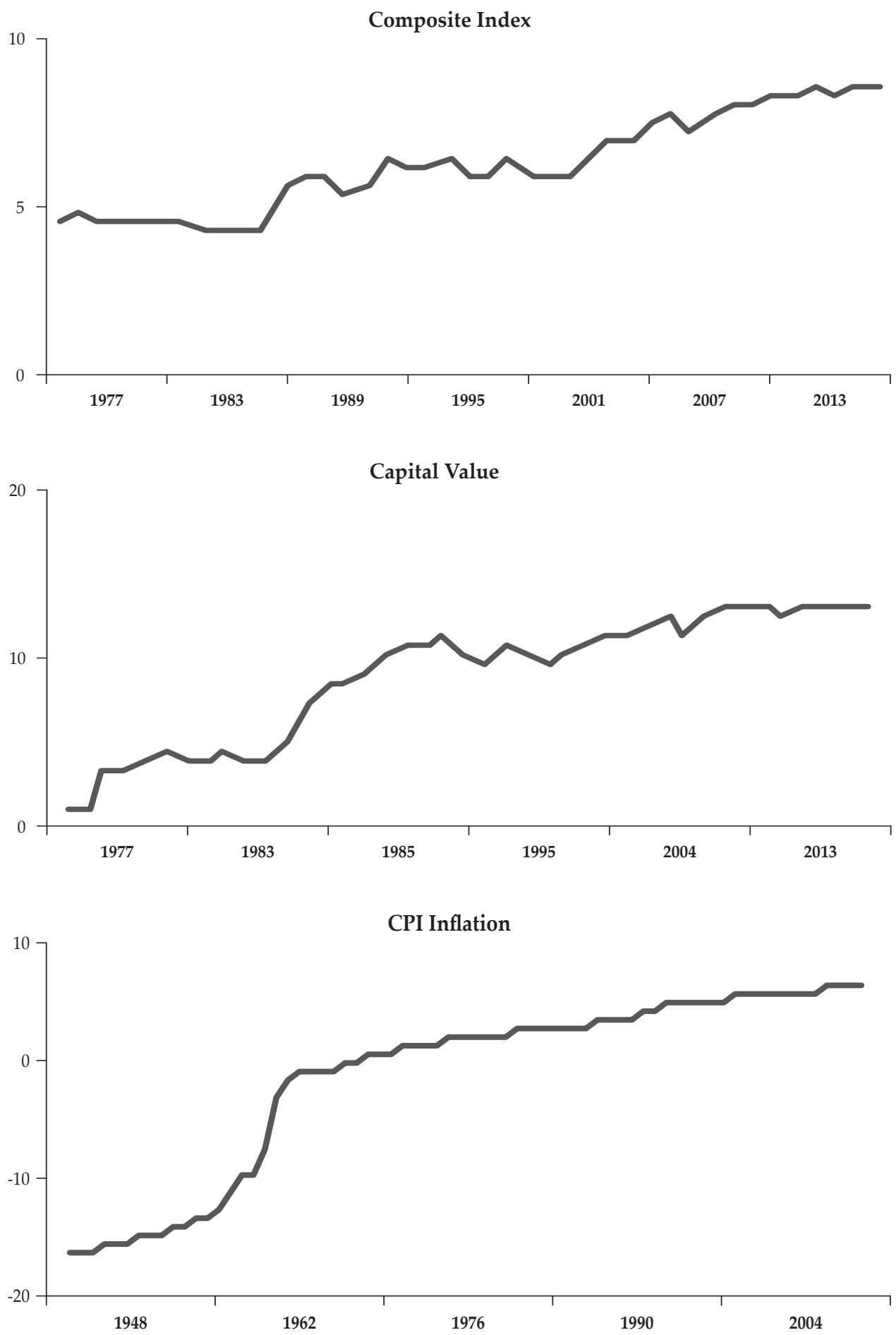
Figure 1. A Plot of Annual Time-Series Data (Continued)
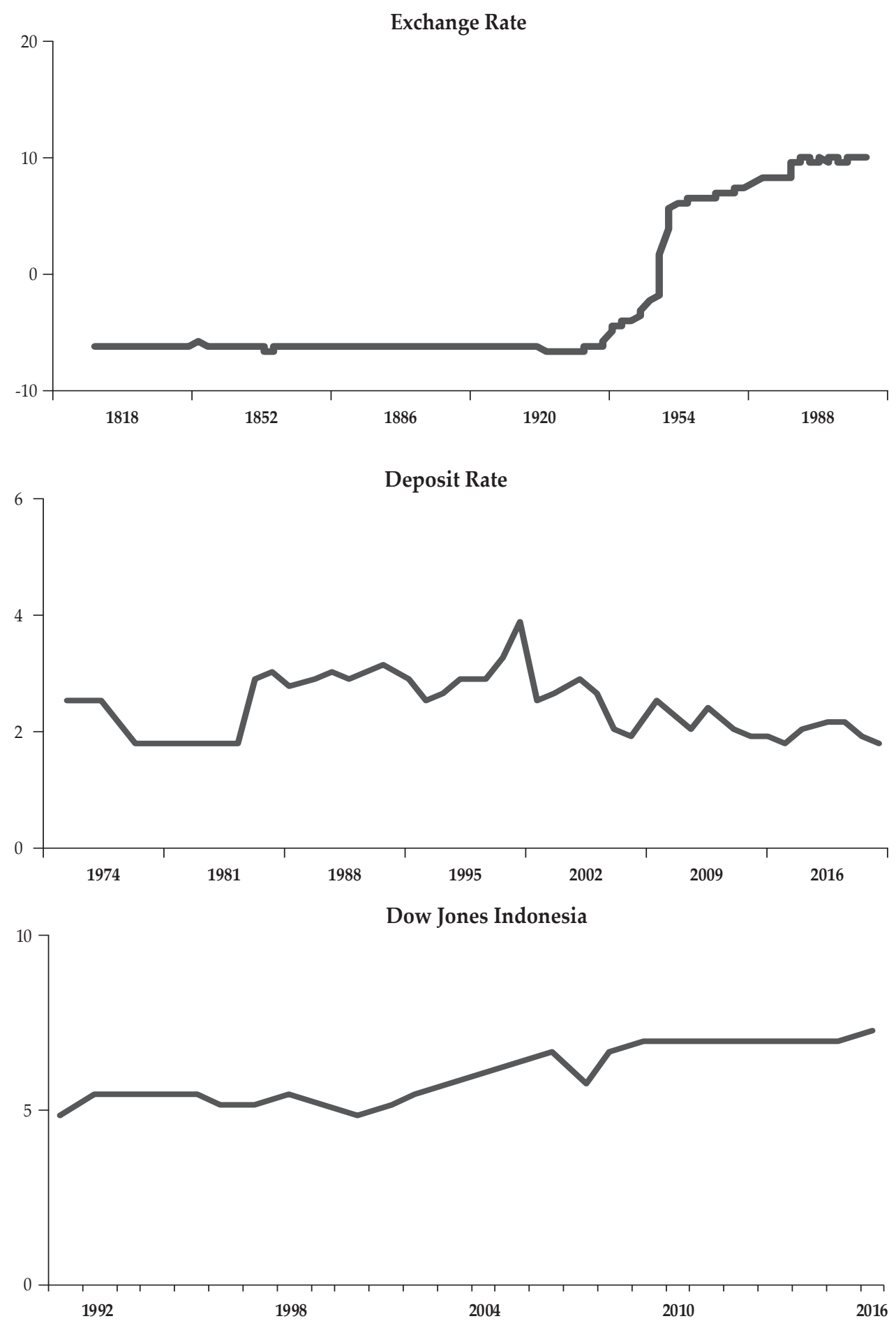
Figure 1. A Plot of Annual Time-Series Data (Continued)
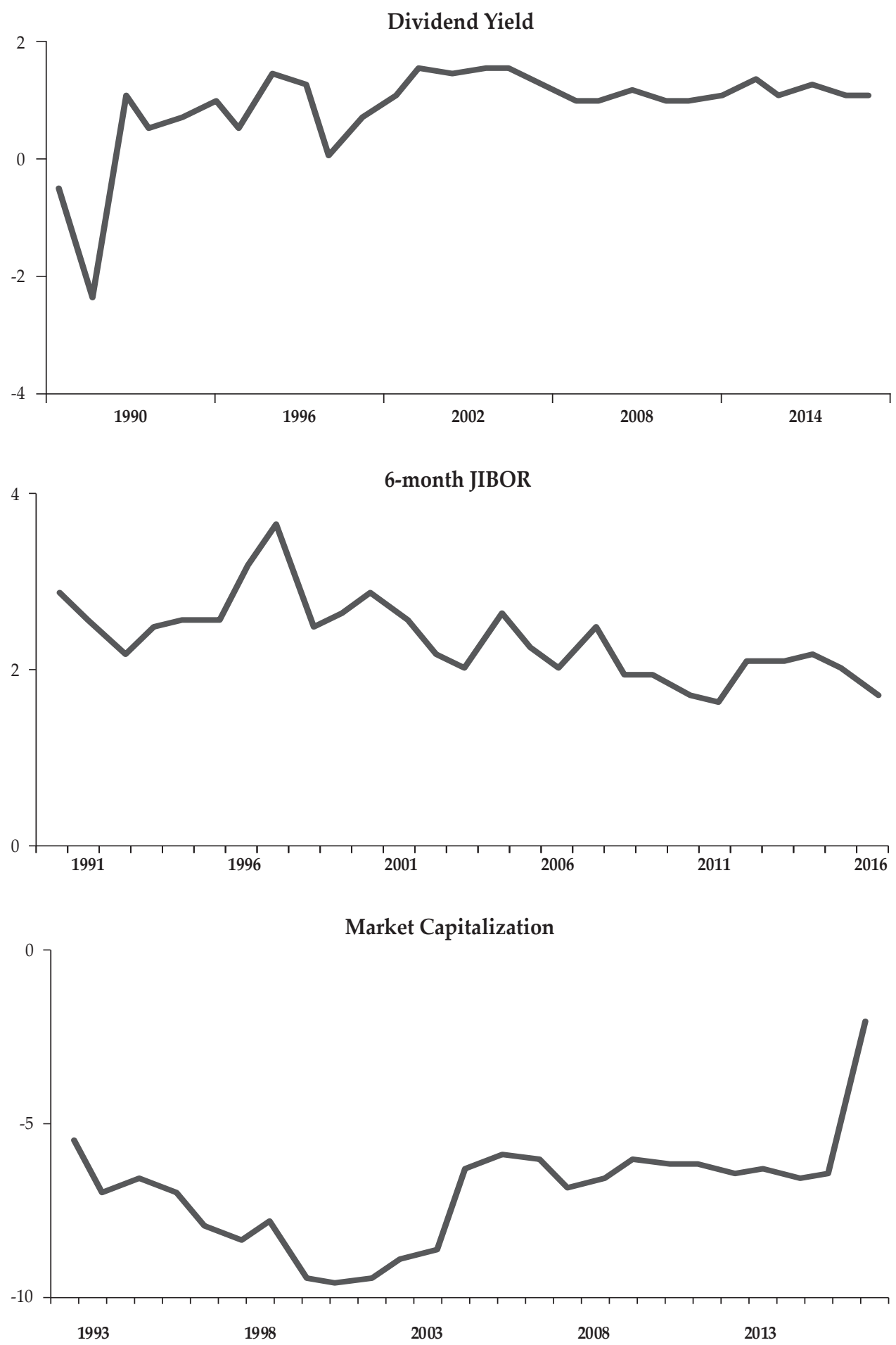
Figure 1. A Plot of Annual Time-Series Data (Continued)

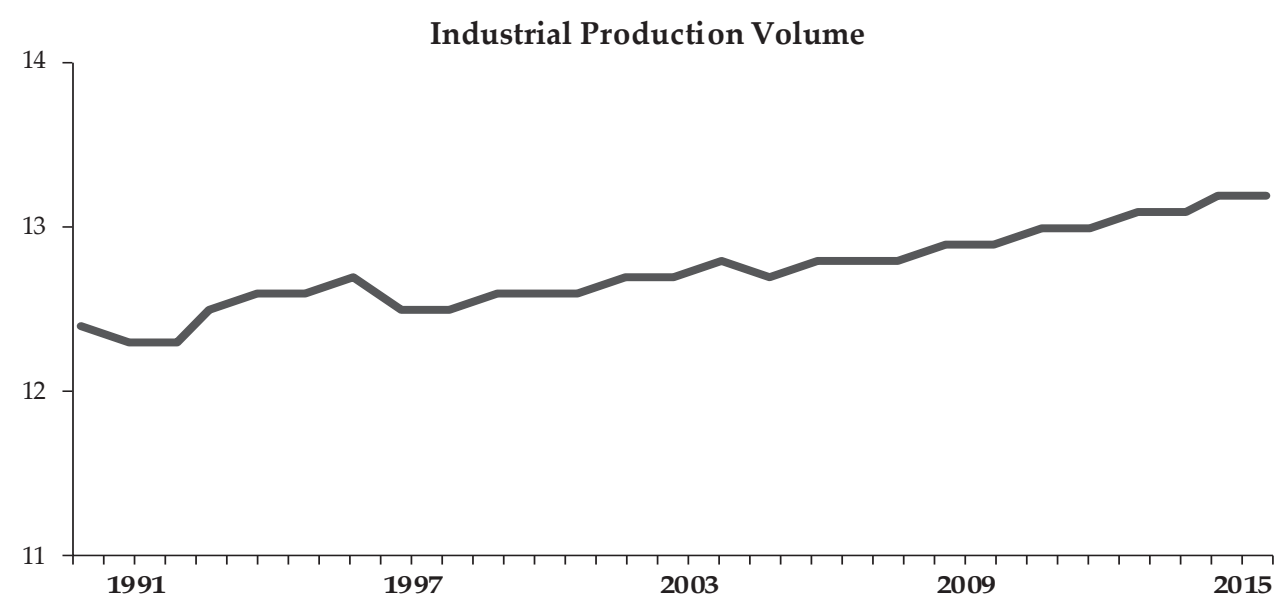

1-month JIBOR

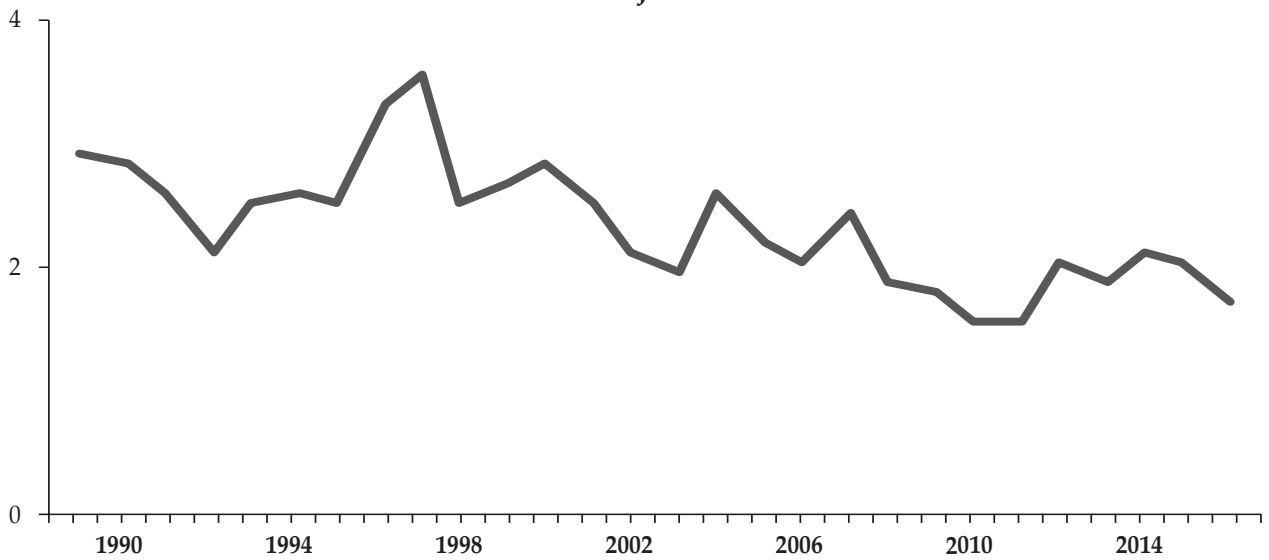

3-month JIBOR

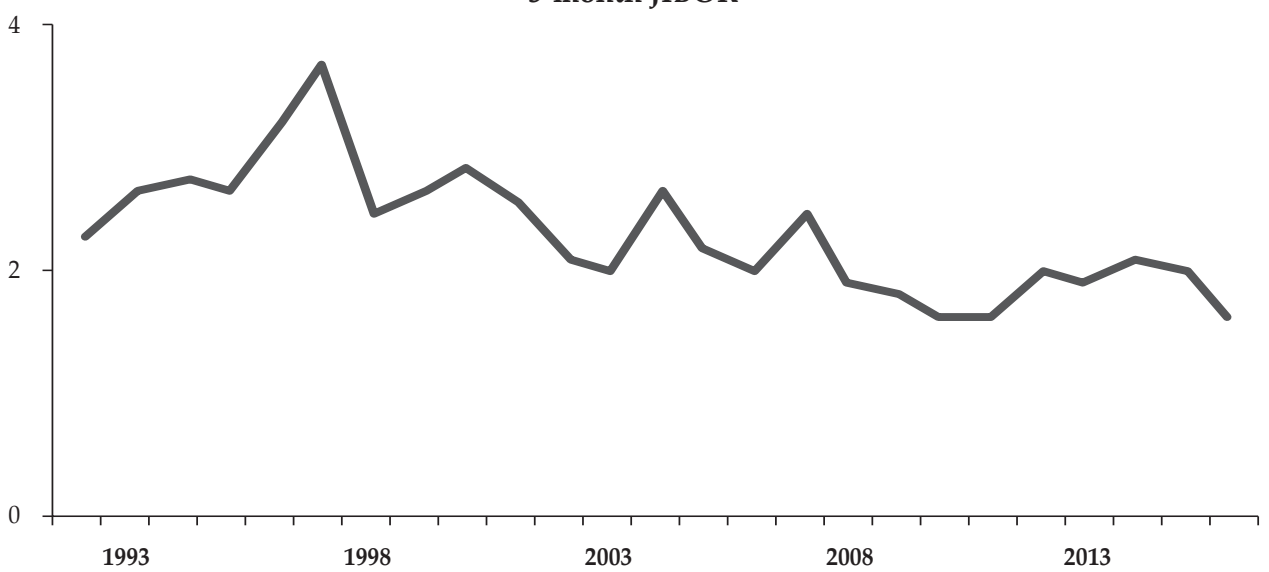


Figure 1. A Plot of Annual Time-Series Data (Continued)

Price to Earning Ratio

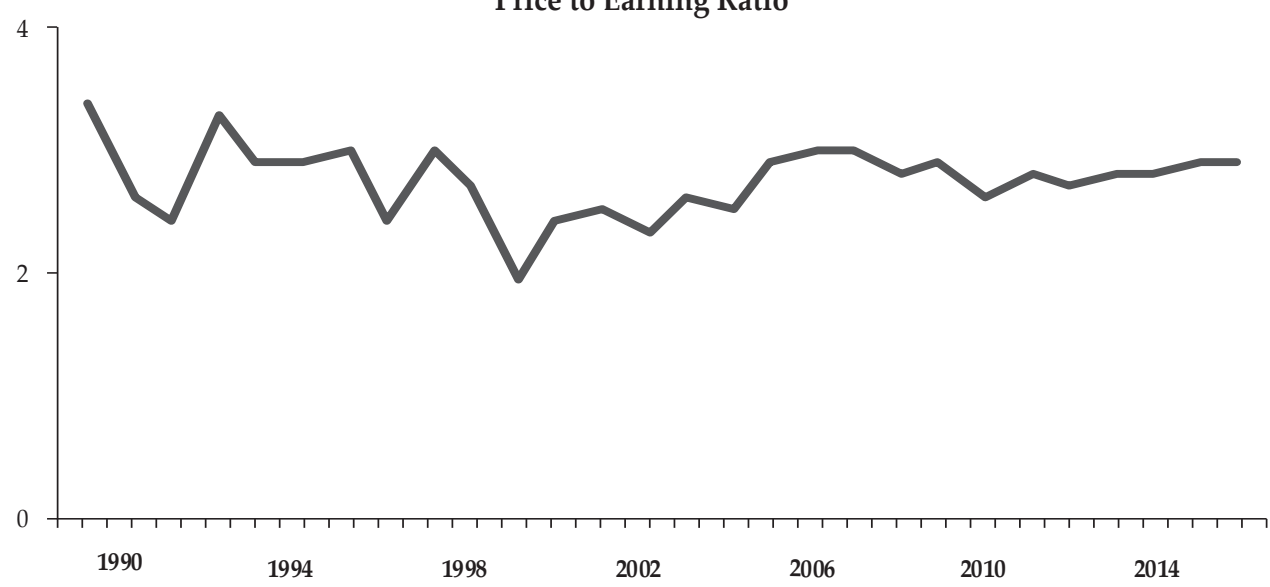

Lending Rate

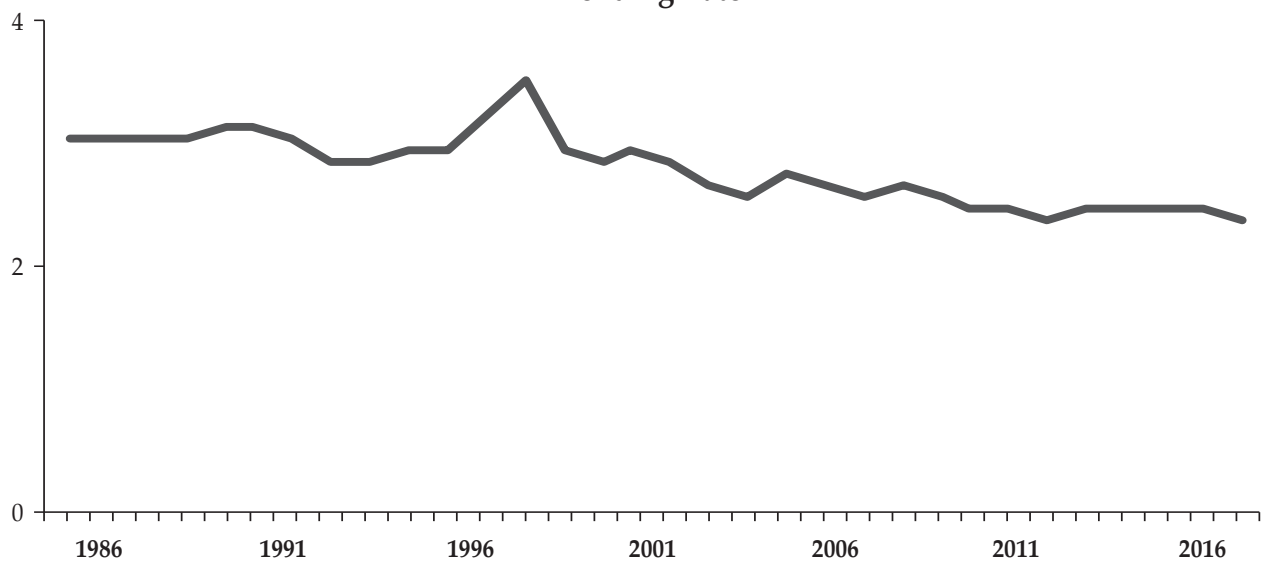

12-month JIBOR

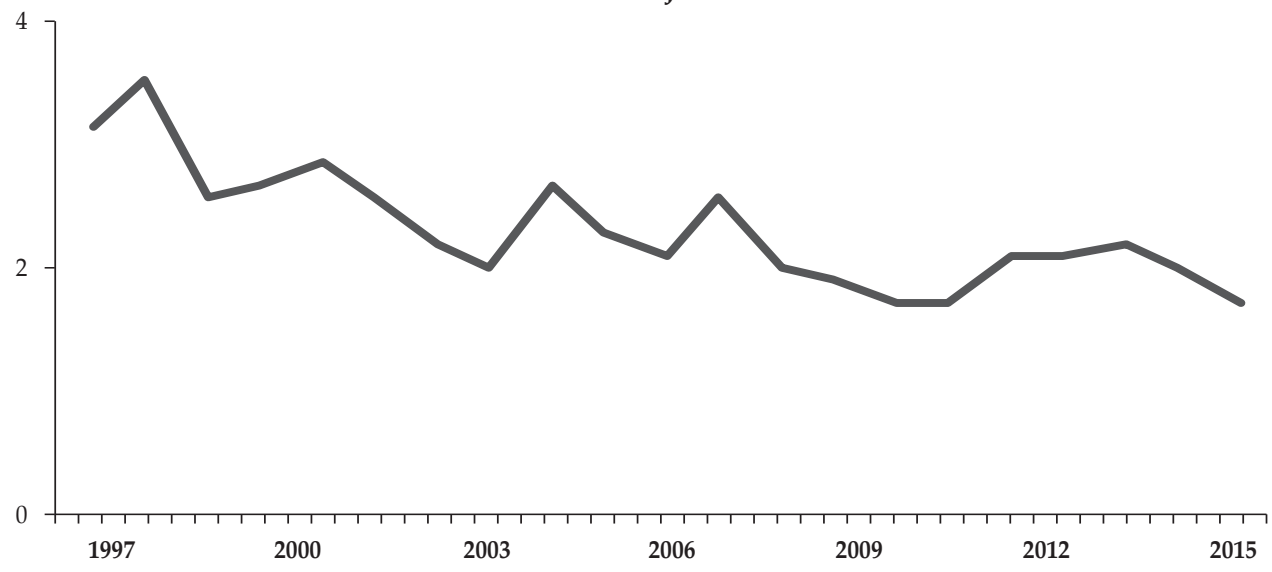


Figure 1. A Plot of Annual Time-Series Data (Continued)

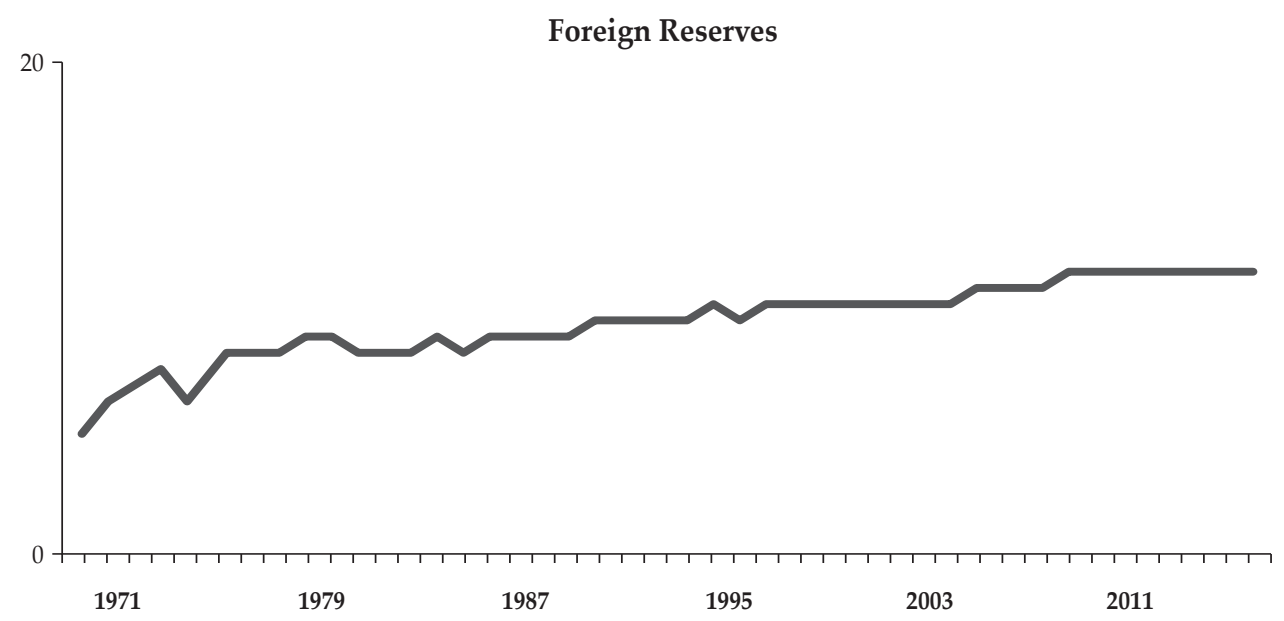

Real GDP
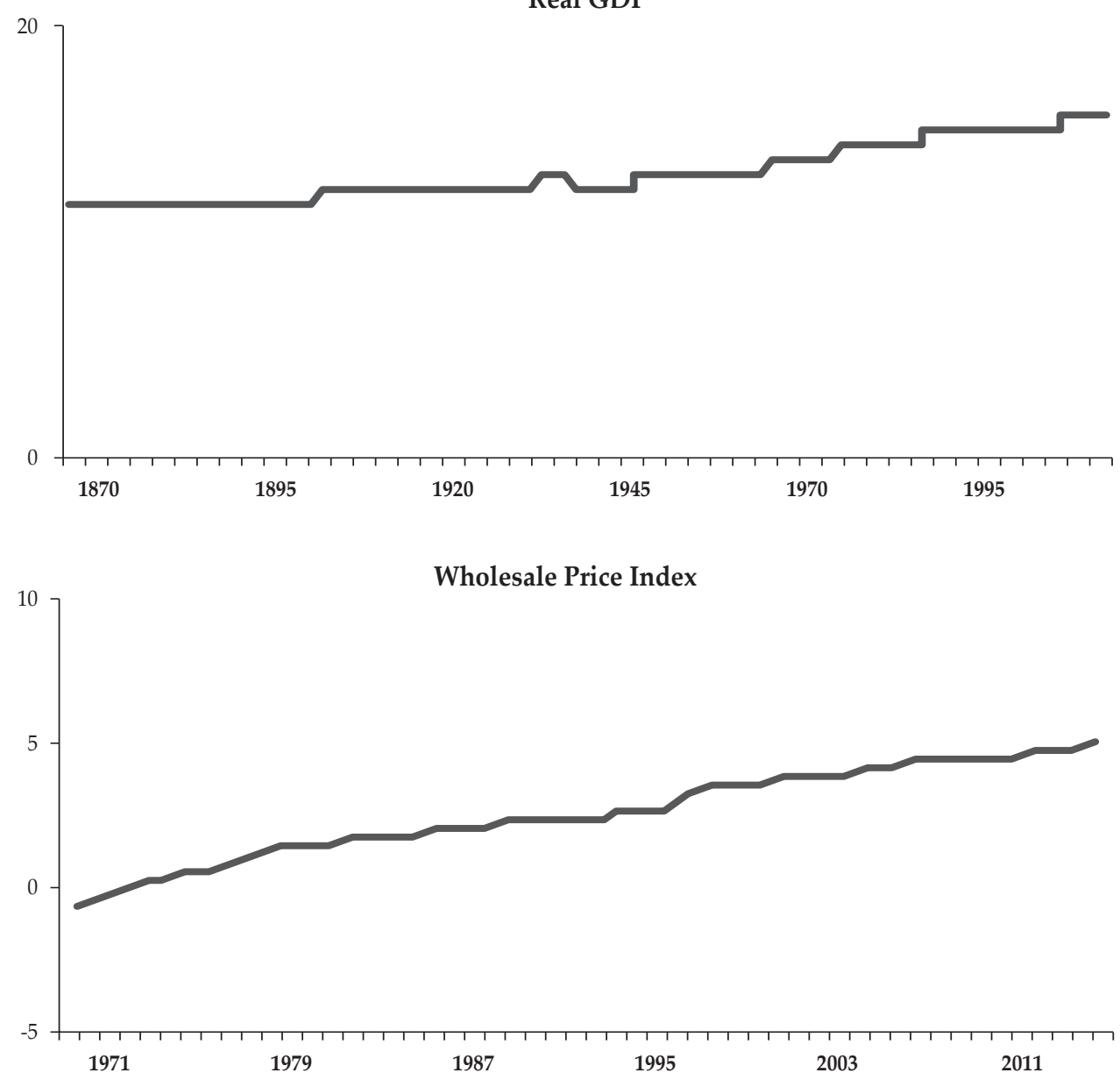
Figure 1. A Plot of Annual Time-Series Data (Continued)
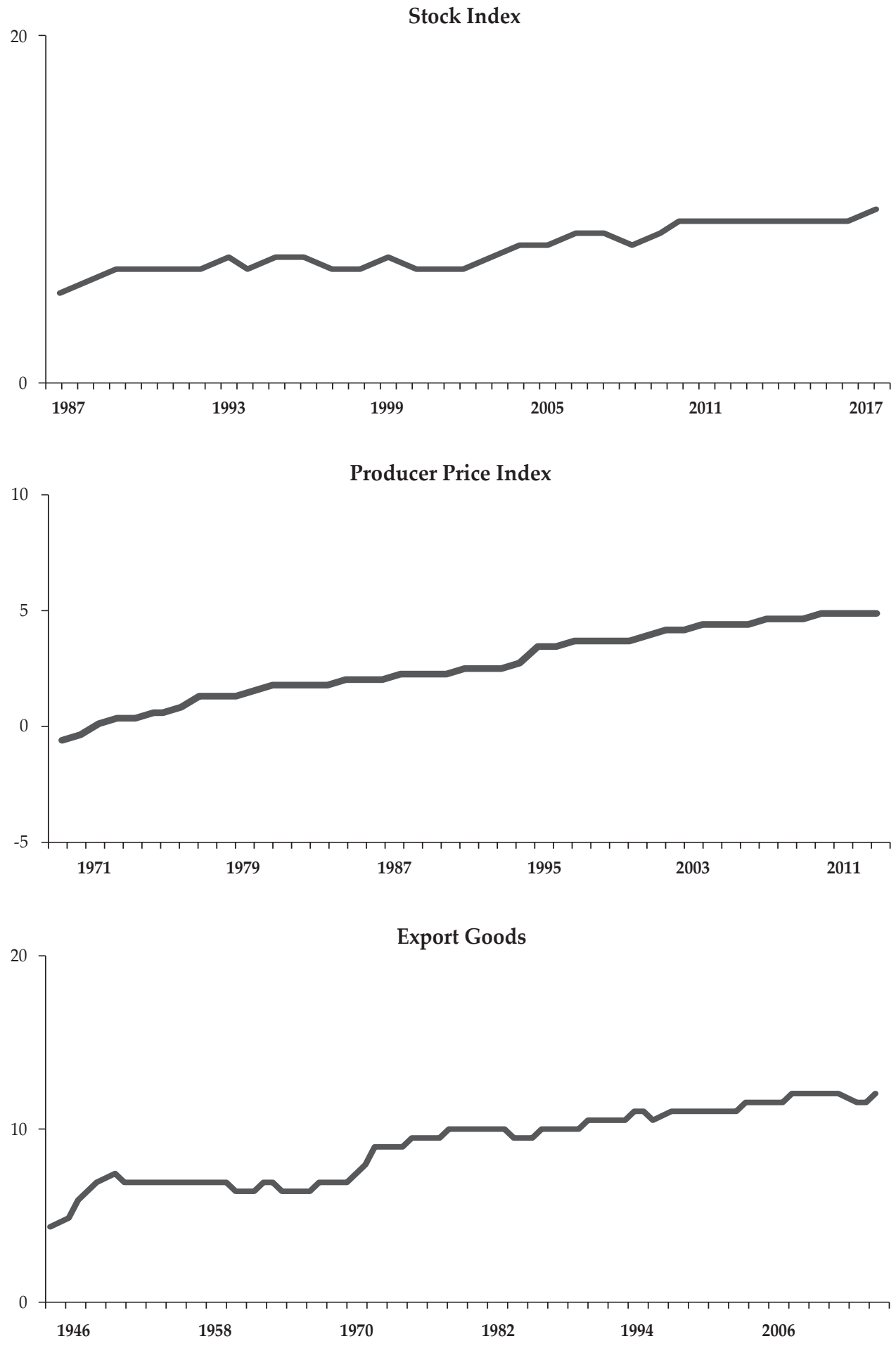
Figure 1. A Plot of Annual Time-Series Data (Continued)
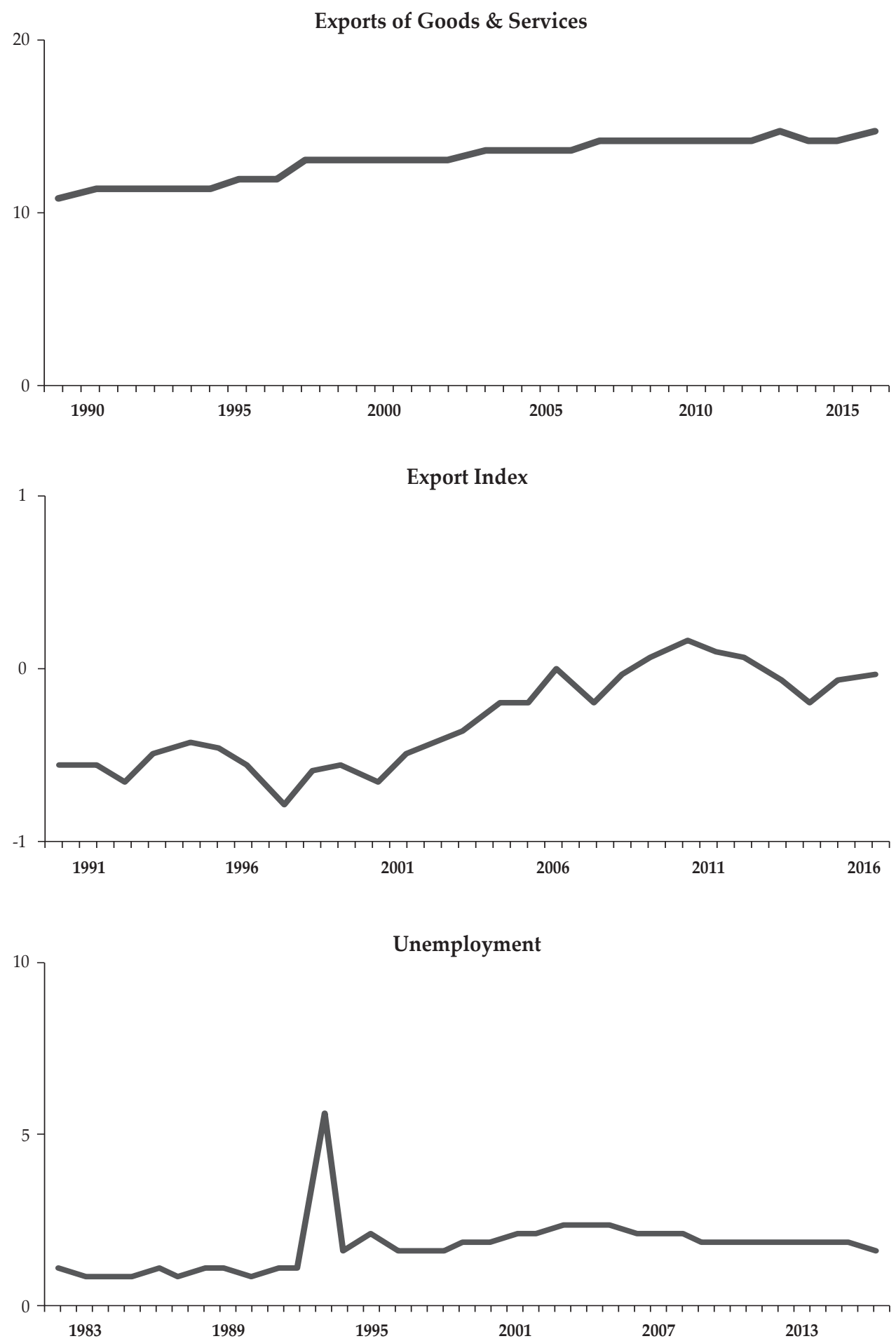
Figure 1. A Plot of Annual Time-Series Data (Continued)

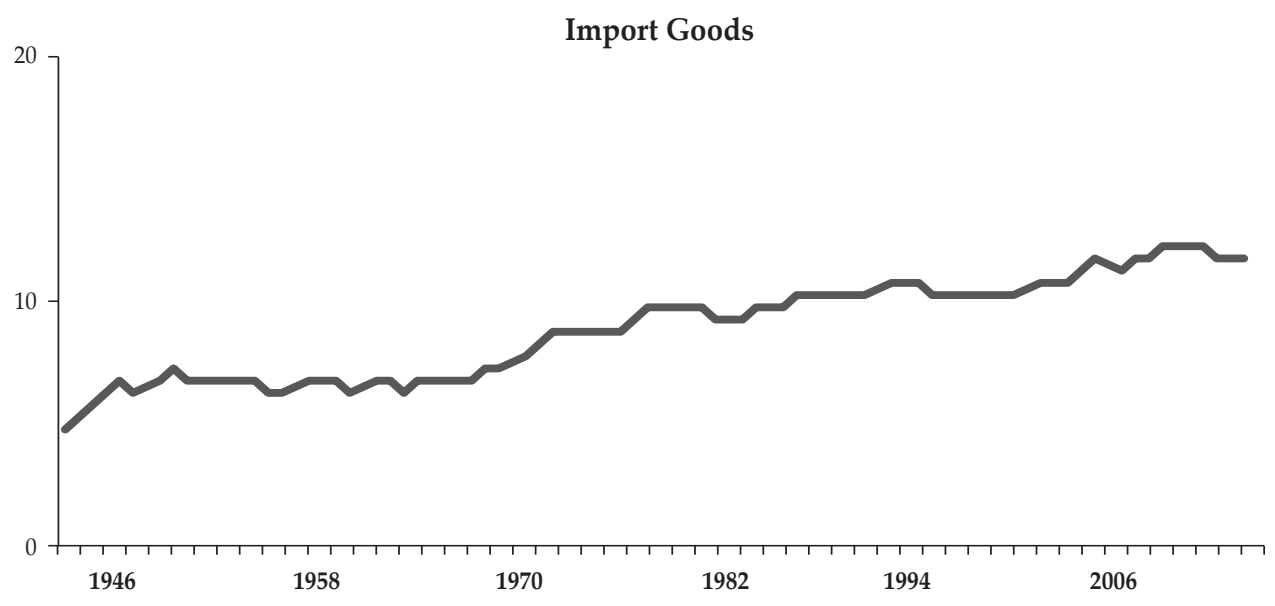

Import Index
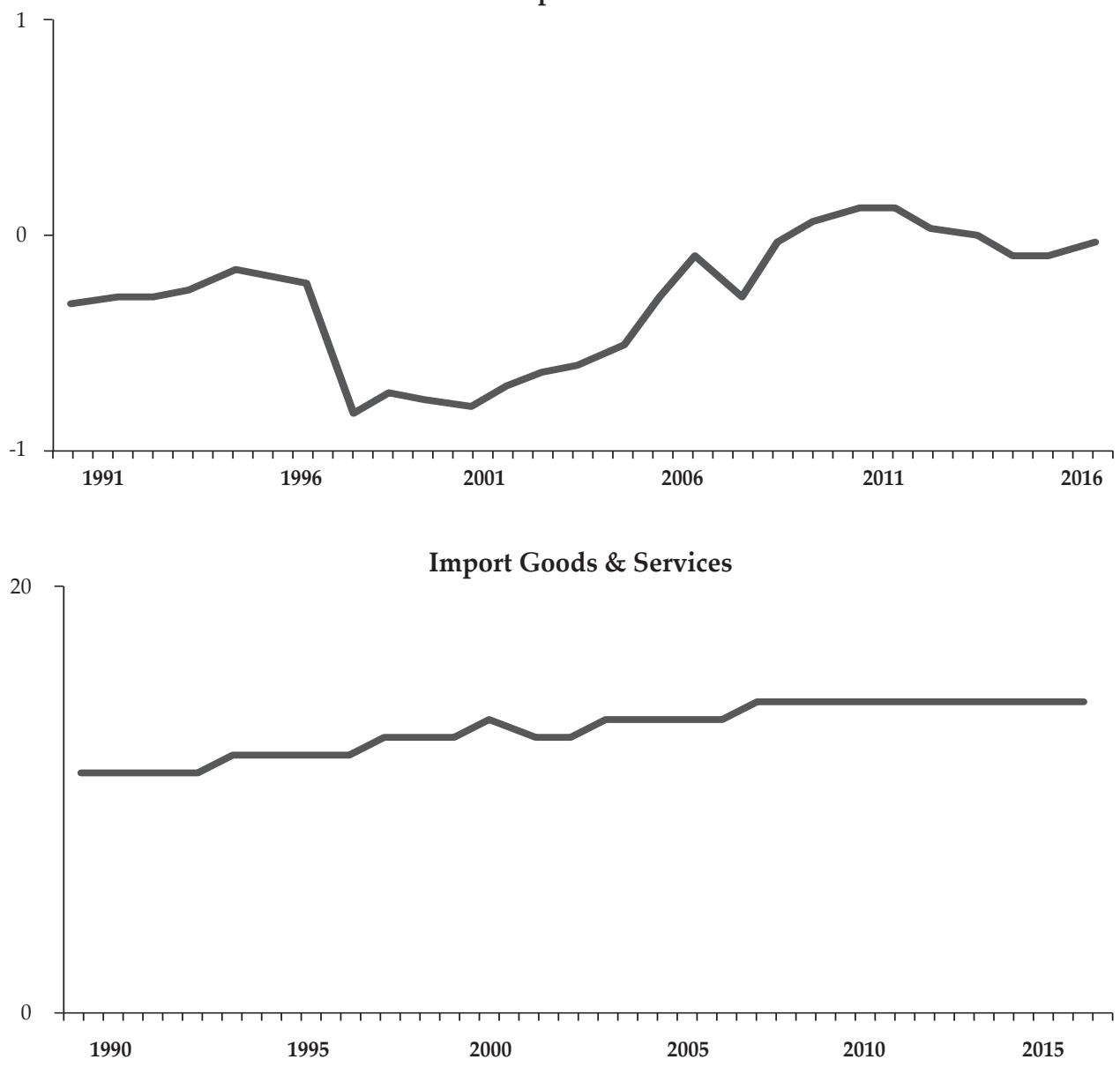
Figure 1. A Plot of Annual Time-Series Data (Continued)
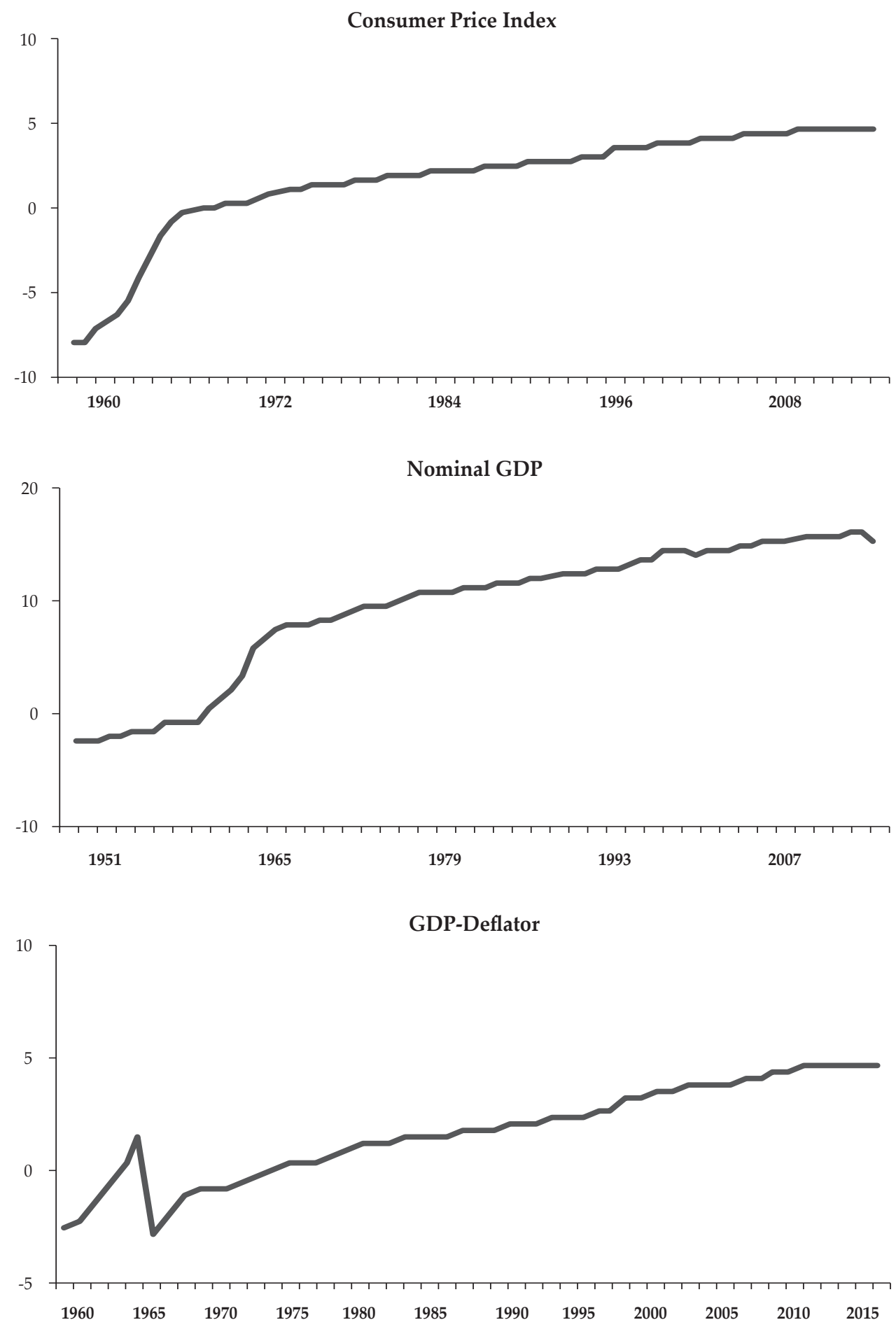
Figure 1. A Plot of Annual Time-Series Data (Continued)
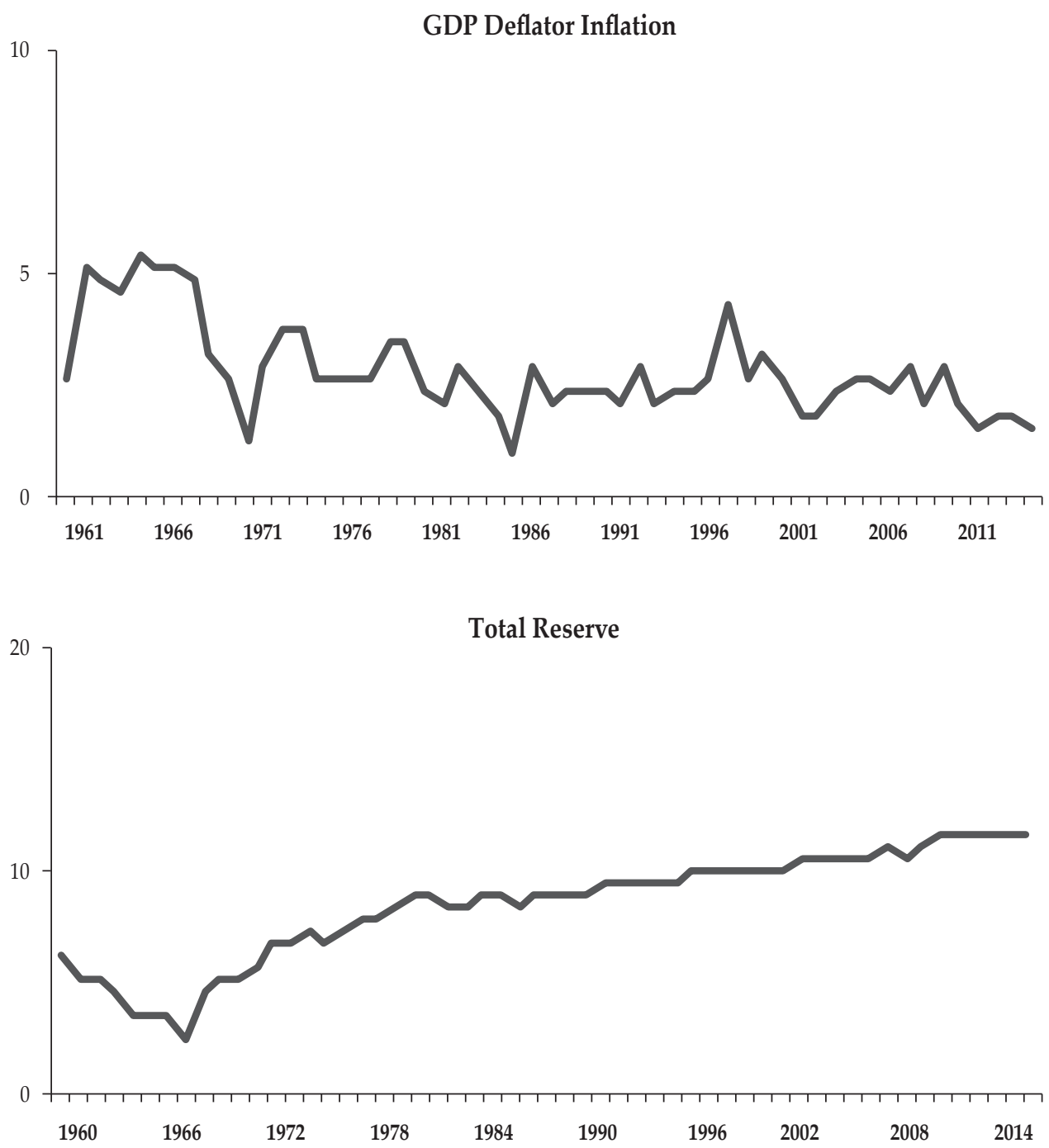

The Narayan and Popp (2010) test results for monthly data are reported in Table 4. We document that regardless of the type of model specification (i.e., Model 1 or Model 2), the unit root null hypothesis with monthly data is rejected for business confidence index, capital value traded, cash return index, consumer confidence index, exchange rate, 1- and 3-month interbank interest rate, industrial production (volume), lending rate, M1, price-earnings ratio, and foreign reserves. In total, therefore, we discover that the unit root hypothesis can be rejected in $13 / 31$ monthly series, equivalent to $42 \%$ of the time-series data on hand. 
Table 4.

Unit Root Results for Monthly Data

This table shows Narayan and Popp (2010) unit root results for monthly data. Columns 3 and 4 show the sample period and the corresponding number of observations (T). We refer to Table 3 of Narayan and Popp (2010) for critical values for unknown break dates. Models 1 and 2 are two models for testing unit root. Model 1 (see Column 5) allows for two breaks in level and the Model 2 allows for two breaks in level as well as slope (see Column 6). The true break dates are denoted by TB1 and TB2; k represents the optimal lag length; and ${ }^{* * *},{ }^{* *}$, and ${ }^{*}$ indicate that the unit root null hypothesis is rejected at the $1 \%, 5 \%$, and $10 \%$ levels of significance, respectively.

\begin{tabular}{|c|c|c|c|c|c|c|c|c|c|c|c|}
\hline \multirow{2}{*}{\multicolumn{2}{|c|}{ No. Series }} & \multirow[b]{2}{*}{ Sample } & \multirow[b]{2}{*}{$\mathrm{T}$} & \multicolumn{4}{|l|}{ M1 } & \multicolumn{2}{|l|}{ M2 } & \multirow[b]{2}{*}{ TB2 } & \multirow[b]{2}{*}{$\mathrm{k}$} \\
\hline & & & & T-stat & TB1 & TB2 & $\mathrm{k}$ & T-stat & TB1 & & \\
\hline 1 & Bond Yield, 3 Year & 2009:05-2018:06 & 110 & -3.796 & 2011:08 & 2013:05 & 4 & -4.306 & 2011:08 & 2013:05 & 4 \\
\hline 2 & Bond Yield, 5 Year & 2009:05-2018:06 & 110 & -3.480 & 2013:05 & 2013:09 & 0 & -3.062 & 2013:05 & 2013:10 & 0 \\
\hline 3 & Bond Yield, 10 Year & 2009:05-2018:06 & 110 & -3.711 & 2011:12 & 2013:05 & 0 & -4.123 & 2013:05 & 2013:10 & 3 \\
\hline 4 & Business Confidence Index & 2002:01-2017:12 & 190 & $-5.235^{* * *}$ & 2006:08 & 2006:11 & 3 & $-5.170^{* *}$ & 2006:08 & 2006:12 & 3 \\
\hline 5 & Capital Value Traded & 1990:01-2018:05 & 341 & -2.639 & 1997:07 & 1998:07 & 2 & $-5.520^{* * *}$ & 1997:07 & 2008:09 & 5 \\
\hline 6 & Cash Return Index & 1989:12-2018:06 & 343 & $-6.238^{* * *}$ & 1997:07 & 1997:10 & 4 & -3.535 & 1997:07 & 1998:09 & 4 \\
\hline 7 & Composite Index & 1983:03-2018:06 & 424 & -3.026 & 1997:07 & 2008:09 & 1 & -3.613 & 1997:07 & 2008:09 & 1 \\
\hline 8 & $\begin{array}{l}\text { Consumer Confidence } \\
\text { Index }\end{array}$ & 2001:04-2017:12 & 201 & $-4.099^{*}$ & 2004:09 & 2006:12 & 1 & -4.585 & 2004:09 & 2006:12 & 1 \\
\hline 9 & CPI Inflation & 1967:01-2018:06 & 618 & $-5.400^{* * *}$ & 1998:01 & 2005:09 & 4 & $-6.085^{* * *}$ & 1998:01 & 2005:09 & 4 \\
\hline 10 & Deposit Rate & 1974:04-2016:07. & 508 & -2.882 & 1984:02 & 1997:07 & 3 & -3.451 & 1984:02 & 1997:07 & 3 \\
\hline 11 & Dividend Yield & 1990:11-2018:06 & 332 & -3.339 & 1999:06 & 2000:03 & 0 & -3.648 & 1999:06 & 2000:03 & 0 \\
\hline 12 & Exchange Rate & 1876:01-2018:06 & 1710 & $-6.105^{* * *}$ & $1960: 07$ & 1963:12 & 4 & $-4.498^{*}$ & 1960:07 & 1963:12 & 4 \\
\hline 13 & Dow Jones Stock Index & 1992:01-2018:06 & 318 & -2.690 & 1998:07 & 2008:09 & 0 & -3.675 & 1998:07 & 2008:09 & 0 \\
\hline 14 & Export Goods & 1961:01-2018:05 & 689 & -2.014 & 1974:01 & 1977:02 & 4 & -1.951 & 1974:01 & 1977:02 & 4 \\
\hline 15 & Export Index & 1991:01-2018:05 & 329 & -2.072 & 1997:12 & 2008:10 & 5 & -3.703 & 1997:12 & 2008:10 & 5 \\
\hline 16 & $\begin{array}{l}\text { GFD Market Capitalisation } \\
\text { of GDP }\end{array}$ & 1995:01-2018:05 & 281 & -1.241 & 2004:04 & 2005:11 & 0 & -1.825 & 2004:04 & 2005:11 & 0 \\
\hline 17 & Import Goods & 1960:01-2018:06 & 701 & -2.363 & 1978:03 & 1986:11 & 3 & -3.067 & 1978:03 & 1986:11 & 3 \\
\hline 18 & Import Index & 1991:01-2018:05 & 329 & -2.457 & 1997:12 & 1998:04 & 5 & -1.792 & 1997:12 & 1998:06 & 5 \\
\hline 19 & $\begin{array}{l}\text { Indonesia } 1 \text { Month } \\
\text { Interbank Interest Rate } \\
\text { (JIBOR) }\end{array}$ & 1990:01-2018:06 & 342 & -3.791 & 1997:07 & 1997:10 & 5 & $-4.559^{*}$ & 1997:07 & 1998:01 & 4 \\
\hline 20 & $\begin{array}{l}\text { Indonesia } 3 \text { Month } \\
\text { Interbank Interest Rate } \\
\text { (JIBOR) }\end{array}$ & 1993:12-2018:06 & 295 & -2.566 & 1999:04 & 1999:06 & 0 & $-4.449^{*}$ & 1999:05 & 2005:07 & 5 \\
\hline 21 & $\begin{array}{l}\text { Indonesia } 6 \text { Month } \\
\text { Interbank Interest Rate } \\
\text { (JIBOR) }\end{array}$ & 1991:01-2018:06 & 330 & -3.102 & 1997:08 & 1999:05 & 5 & -3.032 & 1997:08 & 1998:04 & 5 \\
\hline 22 & $\begin{array}{l}\text { Indonesia } 12 \text { Month } \\
\text { Interbank Interest Rate } \\
\text { (JIBOR) }\end{array}$ & 1997:03-2018:06 & 256 & -3.423 & 2005:07 & 2008:09 & 5 & -4.373 & 2005:07 & 2008:09 & 5 \\
\hline 23 & $\begin{array}{l}\text { Industrial Production } \\
\text { Volume }\end{array}$ & 1991:12-2018:04 & 317 & $-4.408^{*}$ & 1999:01 & 2003:11 & 4 & $-6.984^{* * *}$ & 1997:12 & 2003:11 & 4 \\
\hline 24 & $\begin{array}{l}\text { Jakarta Stock Exchange } \\
\text { Islamic Index }\end{array}$ & 2000:07-2018:06 & 216 & -2.981 & 2004:10 & 2008:09 & 3 & -4.026 & 2008:02 & 2008:09 & 0 \\
\hline 25 & $\begin{array}{l}\text { Lending Rate for Working } \\
\text { Capital }\end{array}$ & 1986:03-2016:08 & 366 & $-4.534^{* *}$ & 1997:07 & 1998:02 & 5 & $-5.126^{* *}$ & 1997:07 & 1998:05 & 5 \\
\hline 26 & M1-Money Supply & 2008:01-2018:04 & 124 & $-4.691^{* *}$ & 2010:11 & 2011:11 & 3 & $-5.840^{* * *}$ & 2011:11 & 2013:12 & 0 \\
\hline
\end{tabular}


Table 4.

Unit Root Results for Monthly Data (Continued)

\begin{tabular}{|c|c|c|c|c|c|c|c|c|c|c|c|}
\hline \multirow{2}{*}{\multicolumn{2}{|c|}{ No. Series }} & \multirow[b]{2}{*}{ Sample } & \multirow[b]{2}{*}{$\mathrm{T}$} & \multicolumn{4}{|l|}{ M1 } & \multicolumn{4}{|l|}{ M2 } \\
\hline & & & & T-stat & TB1 & TB2 & $\mathrm{k}$ & $T$-stat & TB1 & TB2 & $\mathrm{k}$ \\
\hline 27 & M2-Money Supply & 2008:01-2018:04 & 124 & -1.627 & 2010:11 & 2011:11 & 4 & -1.848 & 2010:11 & 2011:11 & 4 \\
\hline 28 & Price to Earnings Ratio & 1990:01-2018:06 & 342 & $-4.719^{* *}$ & 1998:09 & 2008:12 & 1 & $-5.118^{* *}$ & 1998:09 & 2008:12 & 1 \\
\hline 29 & $\begin{array}{l}\text { Producer Price Index } \\
\text { Excluding Oil }\end{array}$ & 1971:01-2016:04 & 544 & -3.374 & 1986:08 & 1997:12 & 5 & -2.136 & 1986:08 & 1997:12 & 5 \\
\hline 30 & Stock Return Index & 1988:01-2018:06 & 366 & -3.277 & 1997:07 & 1998:07 & 1 & -3.530 & 1998:07 & 1998:11 & 0 \\
\hline 31 & $\begin{array}{l}\text { Total Foreign Exchange } \\
\text { Reserves (exclude Gold) }\end{array}$ & 1971:01-2018:06 & 570 & $-6.325^{* * *}$ & 1983:02 & 1990:11 & 5 & -4.018 & 1983:02 & 1987:06 & 5 \\
\hline
\end{tabular}

As a robustness check, we examine annual time-series data. The results from the unit root test are reported in Table 5. With the Model 1, the unit root null is rejected for 12/33 series while with the Model 2, the null is rejected for 9/33 series. Taking both models together, with annual data, a total of 16 series are unit root stationary, meaning the unit root null hypothesis is comfortably rejected. This represents $48 \%$ of the variables.

Table 5.

Unit Root Results for Yearly Data

This table shows Narayan and Popp (2010) unit root results for yearly data. Column 3 and 4 show the sample period and the corresponding number of observations. We refer to the Table 3 of Narayan and Popp (2010) for the critical values for unknown break dates. M1 and M2 are two models for testing unit root. The model M1 (see Column 5) allows for two breaks in level and the model M2 allows for two breaks in level as well as slope (see Column 6). The true break dates are denoted by TB1 and TB2. The $\mathrm{k}$ represents the optimal lag length. ${ }^{* *},{ }^{* *}$, and * indicate the unit root null is rejected, at levels of statistical significance $1 \%, 5 \%$, and $10 \%$, respectively.

\begin{tabular}{|c|c|c|c|c|c|c|c|c|c|c|c|}
\hline \multirow{2}{*}{\multicolumn{2}{|c|}{ No. Series }} & \multirow{3}{*}{$\begin{array}{c}\text { Sample } \\
1977-2017\end{array}$} & \multirow{3}{*}{$\frac{\mathrm{T}}{41}$} & \multirow{2}{*}{$\begin{array}{c}\text { M1 } \\
\text { T-stat }\end{array}$} & \multicolumn{7}{|c|}{ M2 } \\
\hline & & & & & \multirow{2}{*}{$\begin{array}{l}\text { TB1 } \\
1988\end{array}$} & \multirow{2}{*}{$\begin{array}{l}\text { TB2 } \\
1996\end{array}$} & \multirow{2}{*}{$\frac{\mathrm{k}}{2}$} & \multirow{2}{*}{$\begin{array}{l}\text { T-stat } \\
-4.504\end{array}$} & \multirow{2}{*}{$\begin{array}{c}\text { TB1 } \\
1996\end{array}$} & \multirow{2}{*}{$\begin{array}{c}\text { TB2 } \\
1999\end{array}$} & \multirow[t]{2}{*}{$\mathrm{k}$} \\
\hline 1 & Capital Value Traded & & & -4.396 & & & & & & & \\
\hline 2 & Cash Return Index & 1989-2017 & 29 & -0.461 & 1997 & 2000 & 1 & -2.383 & 1997 & 2000 & 0 \\
\hline 3 & Composite Index & $1977-2017$ & 41 & -3.642 & 1987 & 1996 & 0 & -3.322 & 1987 & 1992 & 0 \\
\hline 4 & CPI & 1960-2016 & 57 & -15.732 & 1971 & 1997 & 5 & -9.516 & 1972 & 1997 & 5 \\
\hline 5 & CPI Inflation & 1948-2017 & 70 & -0.274 & 1961 & 1965 & 2 & -5.215 & 1961 & 1965 & 0 \\
\hline 6 & Deposit Rate & 1974-2017 & 44 & -4.881 & 1983 & 1997 & 2 & -2.857 & 1983 & 1998 & 4 \\
\hline 7 & Dividend Yield & $1990-2017$ & 28 & -4.647 & 2001 & 2003 & 5 & -7.136 & 1998 & 2009 & 5 \\
\hline 8 & Dow Jones Stock Index & 1992-2017 & 26 & -4.878 & 1999 & 2007 & 5 & -7.423 & 1999 & 2007 & 0 \\
\hline 9 & Exchange Rate & 1818-2017 & 200 & 1.465 & 1963 & 1966 & 3 & -7.265 & 1952 & 1963 & 1 \\
\hline 10 & Export Goods & $1946-2017$ & 72 & -3.540 & 1973 & 1985 & 0 & -2.282 & 1972 & 1975 & 0 \\
\hline 11 & Export Goods and Services & $1990-2017$ & 28 & -1.780 & 1997 & 2004 & 1 & -2.056 & 1998 & 2004 & 0 \\
\hline 12 & Export Index & 1991-2017 & 27 & -2.627 & 1998 & 2008 & 3 & -3.295 & 1998 & 2007 & 0 \\
\hline 13 & GDP-Deflator Inflation & $1961-2015$ & 55 & -5.610 & 1985 & 1997 & 0 & -6.002 & 1971 & 1997 & 0 \\
\hline 14 & GDP-Deflator & $1960-2015$ & 56 & -4.262 & 1971 & 1997 & 5 & -4.226 & 1971 & 1997 & 4 \\
\hline 15 & $\begin{array}{l}\text { GFD Market Capitalisation } \\
\text { of GDP }\end{array}$ & 1993-2017 & 25 & -0.881 & 2004 & 2007 & 0 & -0.678 & 2004 & 2009 & 0 \\
\hline 16 & Nominal GDP & 1951-2017 & 67 & 2.118 & 1965 & 2001 & 2 & -2.208 & 1965 & 2001 & 1 \\
\hline 17 & Real GDP & 1870-2017 & 148 & -2.168 & 1941 & 1946 & 4 & -4.345 & 1941 & 1948 & 3 \\
\hline
\end{tabular}


Table 5.

Unit Root Results for Yearly Data (Continued)

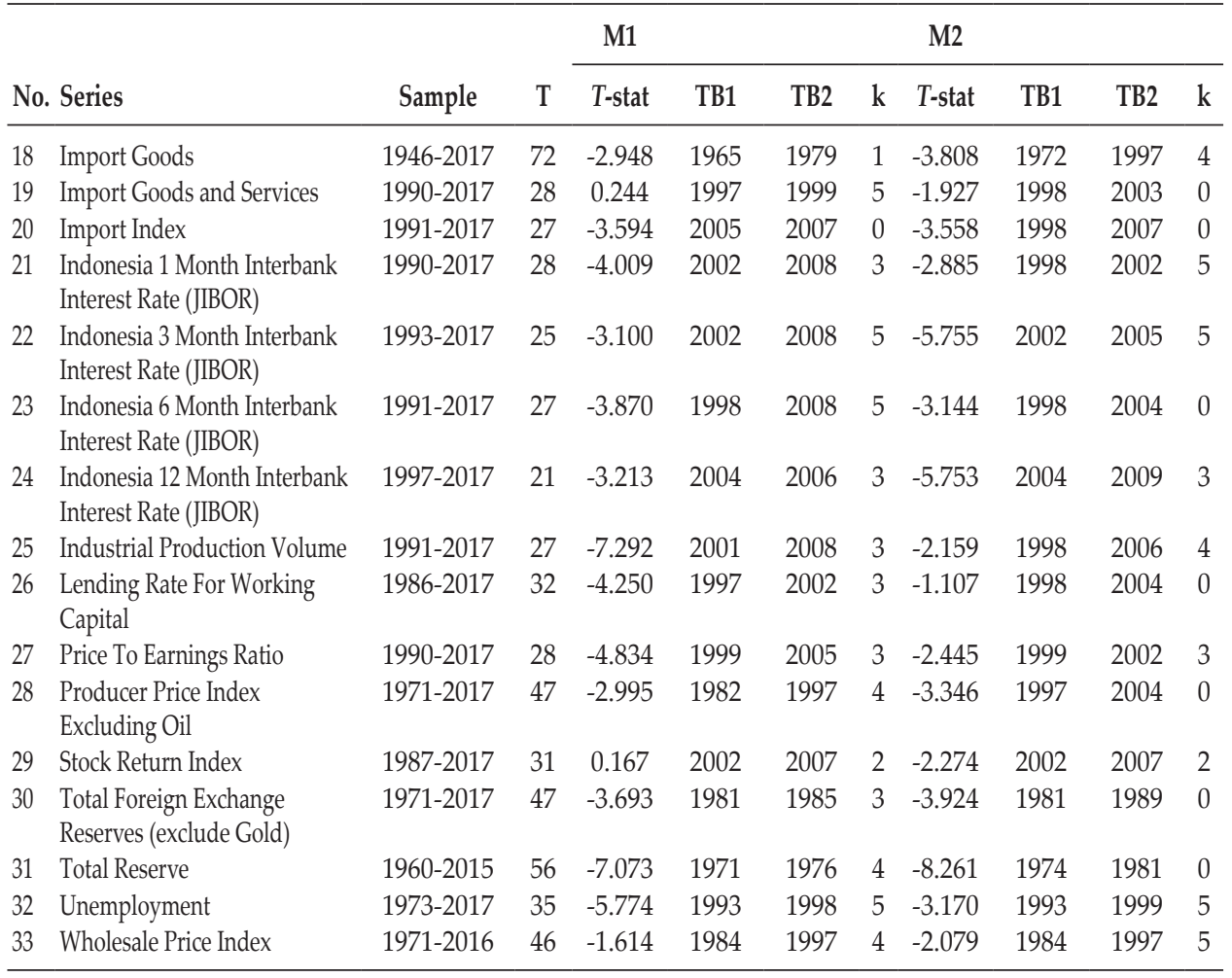

With monthly data, the unit root null hypothesis is rejected for business confidence index, capital value traded, cash return, consumer confidence, CPI inflation, exchange rate, 1- and 3-month interbank interest rate, industrial production (volume), lending rate, M1, price-earnings ratio, and foreign reserves. With annual data, the null is rejected for capital value traded, CPI inflation, deposit rate, dividend yield, Dow Jones stock index, GDP deflator, exchange rate, 3- and 12-month interbank interest rate, industrial production (volume), lending rate, price-earnings ratio, reserves, and unemployment rate. The variables for which the null is rejected regardless of data frequency (in other words, those variables that are stationary in a robust manner) include capital value traded, CPI inflation, exchange rate, industrial production (volume), lending rate, price-earnings ratio, 3 -month interbank interest rate, and foreign reserves. This represents only $24 \%$ of the sample of variables. In other words, data frequency matters to unit root tests and it should be left to policymakers to decide which data frequency is of policy relevance to them in understanding the nature of shocks to time-series data. ${ }^{4}$

\footnotetext{
${ }_{4}$ Some of the break dates relate to obvious events. The monthly CPI inflation break, for instance, corresponds to the period of 2002-2006 when the world oil price increased. In response, the Indonesian government had increased the price of subsidized gasoline by almost two times in 2005 . For yearly CPI inflation data break dates correspond to the period of hyperinflation in Indonesia.
} 


\section{CONCLUDING REMARKS}

This paper examines the URP of macroeconomic time-series data for Indonesia. A total of 33 variables for which sufficient time-series data are available form part of our empirical analysis. We test the hypothesis using the popular Narayan and Popp (2010) unit root test, which allows for two endogenous structural breaks in the data series. Our analysis is based on both annual and monthly time-series data. We find that data frequency is important in understanding URP. First, we show that with annual data, the unit root null hypothesis is rejected in only $48 \%$ of the variables, while with monthly data the number of rejections is equivalent to $42 \%$. The implication here is that there is more evidence of stationarity of variables with annual data than monthly data. Second, across data frequencies, the variables found to be stationary in both data frequencies are capital value traded, CPI inflation, exchange rate, industrial production (volume), lending rate, price-earnings ratio, 3-month interbank interest rate, and foreign reserves. This represents only $24 \%$ of the sample of variables. The implication is that, for these variables, shocks have only a short-term or temporary effect.

Three policy implications emerge from our analysis. First, for policy purposes, it matters whether one uses annual or monthly data. It seems there are more cases of stationary variables with annual data than monthly data, suggesting that more data at annual frequency will be relevant for understanding short-run effects. The second implication relates to forecasting. In most cases, for policy purposes, practitioners need to forecast inflation, exchange rate, and short-term interest rate. These variables for Indonesia are stationary, meaning standard forecasting models that require the dependent variable (variable to be forecast) to be stationary are ideal for forecasting these variables. The third implication concerns the importance of structural breaks. The results described in this paper make clear that structural breaks characterize Indonesia's macroeconomic data. Therefore, it would be costly to ignore breaks in data when econometric modeling, including forecasting, is the subject of research.

\section{REFERENCES}

Amir, H., Asafu-Adjaye, J., and Ducpham, T. (2013). The impact of the Indonesian income tax reform: A CGE analysis. Economic Modelling, 31, 492-501.

Aslanidis, N., and Fountas, S. (2014). Is real GDP stationary? Evidence from a panel unit root test with cross-sectional dependence and historical data. Empirical Economics, 46, 101-108.

Cavaliere, G., and Xu, F. (2014). Testing for unit roots in bounded time series. Journal of Econometrics, 178, 259-272.

Chambers, M. (2015). Testing for a unit root in a near integrated model with skipsampled data. Journal of Time Series Analysis, 36, 630-649.

Chang, T., Chang, H-L., Chu, H-P., and Su, C-W. (2006). Does PPP hold in African countries? Further evidence based on a highly dynamic non-linear (logistic) unit root test. Applied Economics, 38, 2453-2459.

Chang, T., Yang, M., Liao, H-C., and Lee, C-H. (2007). Hysteresis in unemployment: empirical evidence from Taiwan's region data based on panel unit root tests. Applied Economics, 39, 1335-1340. 
Charles, A., and Darné, O. (2012). Trends and random walks in macroeconomic time series: A reappraisal. Journal of Macroeconomics, 34, 167-180.

Choi, I. (2001). Unit root tests for panel data. Journal of International Money and Finance, 20, 249-272.

Chowdhury, A., Uddin, M., and Anderson, K. (2018). Liquidity and macroeconomic management in emerging markets. Emerging Markets Review, 34, 1-24.

Djuranovil, L. (2014). The Indonesian macroeconomy and the yield curve: A dynamic latent factor approach. Journal of Asian Economics, 34, 1-15.

Drakos, A., Kouretas, G., and Vlamis, P. (2018). Saving, investment and capital mobility in EU member countries: A panel data analysis of the FeldsteinHorioka puzzle. Applied Economics, 50, 3798-3811.

Dutu, R. (2016). Why has economic growth slowed down in Indonesia? An investigation into the Indonesian business cycle using an estimated DSGE model. Journal of Asian Economics, 45, 46-55.

Gil-Alana, L., and Robinson, P. (1997). Testing the unit root and other nonstationarity hypothesis in macroeconomic time series. Journal of Econometrics, 80, 241-268.

Hadiwibowo, Y., and Komatsu, M. (2011). Trilemma and macroeconomic policies under different financial structures in Indonesia. Journal of Asian Economics, 22, 302-310.

Hsing, Y. (2012). Impacts of macroeconomic forces and external shocks on real output for Indonesia. Economic Analysis and Policy, 42, 97-104.

Hurlin, C. (2010). What would Nelson and Plosser find had they used panel unit root tests?. Applied Economics, 42, 1515-1531.

Kappler, M. (2009). Do hours worked contain a unit root? Evidence from panel data. Empirical Economics, 36, 531-555.

Lee, J., and Strazicich, M. (2003). Minimum lagrange multiplier unit root test with two structural breaks. Review of Economics and Statistics, 85, 1082-1089.

Li, H., and Park, S. (2018). Testing for a unit root in a nonlinear quantile autoregression framework. Econometric Reviews, 37, 867-892.

Lucas, A. (1995). An outlier robust unit root test with an application to the extended Nelson-Plosser data. Journal of Econometrics, 66, 153-173.

Lumsdaine, R., and Papell, D. (1997). Multiple trend breaks and the unit root hypothesis. Review of Economics and Statistics, 79, 212-218.

Maslyuk, S., and Smyth, R. (2008). Unit root properties of crude oil spot and futures prices. Energy Policy, 36, 2591-2600.

Narayan, P. K., and Liu, R. (2015). A unit root model for trending time-series energy variables. Energy Economics, 50, 391-402.

Narayan, P. K., Liu, R., and Westerlund, J. (2016). A GARCH model for testing market efficiency. Journal of International Financial Markets Institutions and Money, 41, 121-138.

Narayan, P., and Narayan, S. (2010). Is there a unit root in the inflation rate? New evidence from panel data models with multiple structural breaks. Applied Economics, 42, 1661-1670.

Narayan, P., and Popp, S. (2010). A new unit root test with two structural breaks in level and slope at unknown time. Journal of Applied Statistics, 37, 1425-1438. 
Narayan, P., and Smyth, R. (2005). Structural breaks and unit roots in Australian macroeconomic time series. Pacific Economic Review, 10, 421-437.

Narayan, P. K., and Smyth, R. (2007). Mean reversion versus random walk in G7 stock prices: evidence from multiple trend break unit root tests. Journal of International Financial Markets Institutions and Money, 17, 152-166.

Narayan, P. K., Narayan, S., and Smyth, R. (2008). Are oil shocks permanent or temporary? Panel evidence from crude oil and NGL production in 60 countries. Energy Economics, 30, 919-936.

Narayan, P. K. (2005a). Did Rabuka's military coups have a permanent effect or a transitory effect on tourist expenditure in Fiji: Evidence from Vogelsang's structural break test. Tourism Management, 26, 509-515.

Narayan, P. K. (2005b). The structure of tourist expenditure in Fiji: evidence from unit root structural break tests. Applied Economics, 37, 1157-1161.

Narayan, P. K. (2006a). Are bilateral exchange rates stationary? Evidence from Lagrange multiplier unit root tests for India. Applied Economics, 38, 63-70.

Narayan, P. K. (2006b). Examining structural breaks and growth rates in international health expenditures. Journal of Health Economics, 25, 877-890.

Narayan, P. (2008). Revisiting the US money demand function: an application of the Lagrange multiplier structural break unit root test and the bounds test for a long run relationship. Applied Economics, 40, 897-904.

Nelson, C., and Plosser, C. (1982). Trends and random walks in macroeconomic time series: some evidence and implications. Journal of Monetary Economics, 10, 139-169.

Niang, A., Diagne, A., and Pichery, M. (2011). Exploring the finance-real economy link in U.S.: Empirical evidence from panel unit root and cointegration analysis. Empirical Economics, 40, 253-268.

Perron, P. (1989). The great crash, the oil price shock, and the unit root hypothesis. Econometrica, 57, 1361-1401.

Smyth, R. (2003). Unemployment Hysteresis in Australian States and Territories: Evidence from Panel Data Unit Root Tests. The Australian Economic Review, 36, 181-192.

Sowmya, S., and Prasanna, K. (2018). Yield curve interactions with the macroeconomic factors during global financial crisis among Asian markets. International Review of Economic and Finance, 54, 178-192.

Tanuwidjaja, E., and Choy, K. M. (2006). Central bank credibility and monetary policy in Indonesia. Journal of Policy Modeling, 28, 1011-1022. 


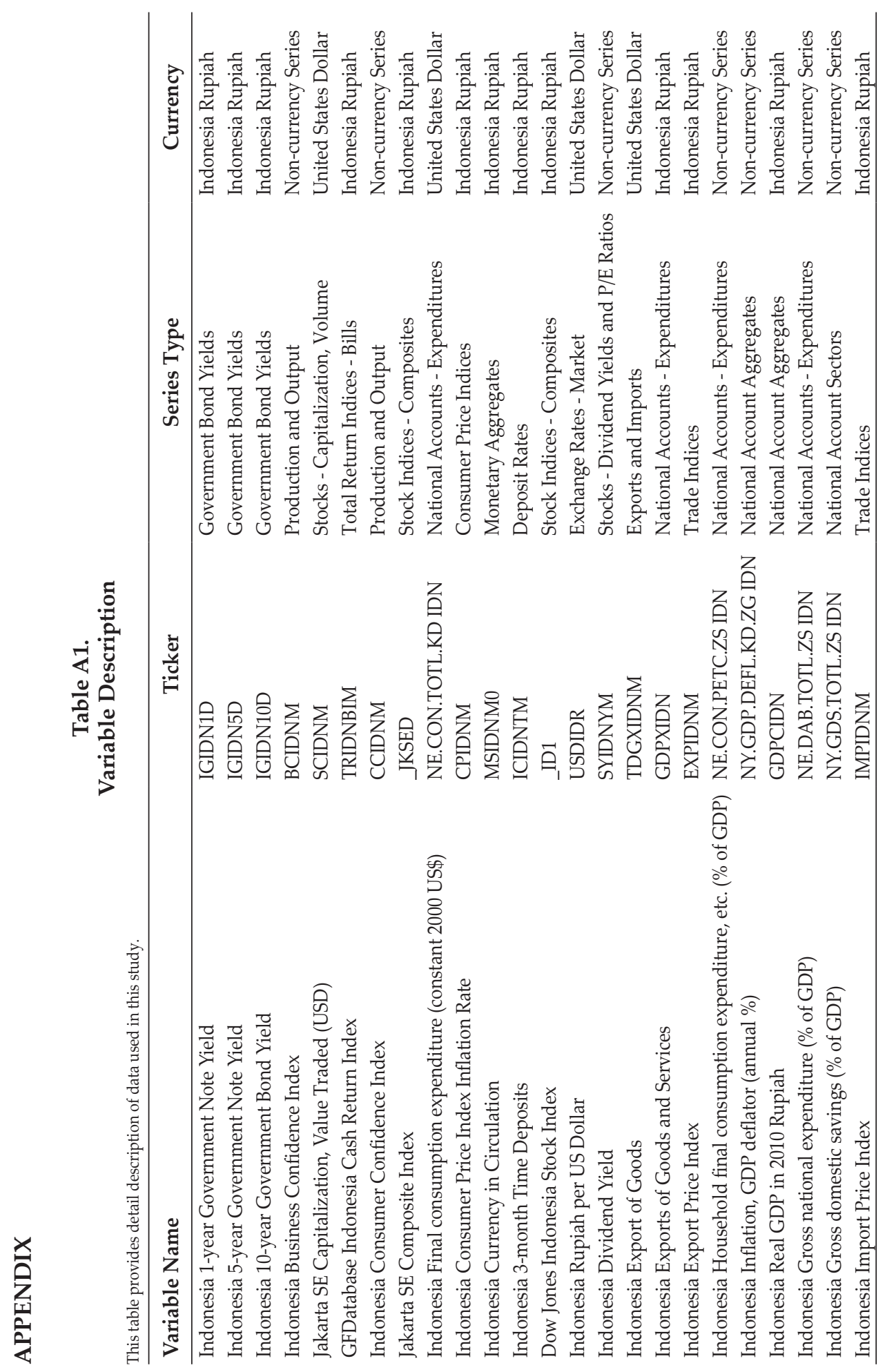




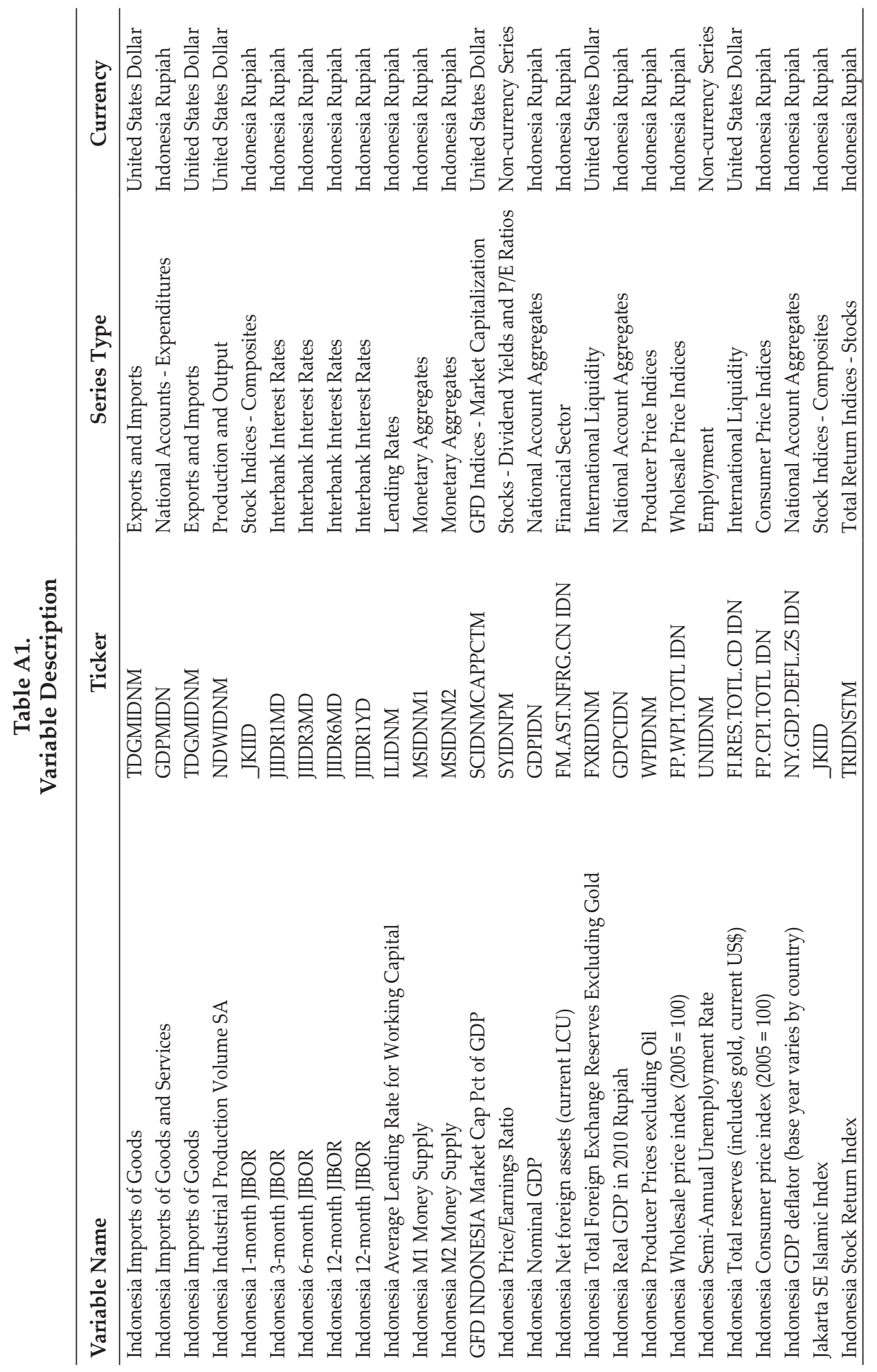

